\title{
Shaping effects on scrape-off layer plasma turbulence: A rigorous validation of three- dimensional simulations against TCV measurements ${ }^{\circledR}$
}

Cite as: Phys. Plasmas 27, 012301 (2020); https://doi.org/10.1063/1.5123451

Submitted: 06 August 2019. Accepted: 11 December 2019. Published Online: 02 January 2020

F. Riva (D), C. K. Tsui (D), J. A. Boedo (D), P. Ricci (D), and TCV Team

COLLECTIONS

EP This paper was selected as an Editor's Pick
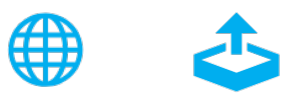

ARTICLES YOU MAY BE INTERESTED IN

Simulation of non-resonant stellarator divertor

Physics of Plasmas 27, 012503 (2020); https://doi.org/10.1063/1.5113907

Simulation of supersonic molecular beam injection fueling into $\mathrm{H}$-mode plasmas on EAST using BOUT++

Physics of Plasmas 27, 012501 (2020); https://doi.org/10.1063/1.5119043

Isotope and plasma size scaling in ion temperature gradient driven turbulence

Physics of Plasmas 26, 120703 (2019); https://doi.org/10.1063/1.5133084

\section{AVs Quantum Science}




\title{
Shaping effects on scrape-off layer plasma turbulence: A rigorous validation of three-dimensional simulations against TCV measurements
}

Cite as: Phys. Plasmas 27, 012301 (2020); doi: 10.1063/1.5123451

Submitted: 6 August 2019 - Accepted: 11 December 2019 .

Published Online: 2 January 2020

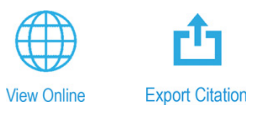

F. Riva, ${ }^{1,2, a)}$ (D) C. K. Tsui, ${ }^{1,3}$ (D) J. A. Boedo, ${ }^{3}$ (D) P. Ricci, (D) and TCV Team ${ }^{\text {b) }}$

\begin{abstract}
AFFILIATIONS
'École Polytechnique Fédérale de Lausanne (EPFL), Swiss Plasma Center (SPC), CH-1015 Lausanne, Switzerland

${ }^{2}$ United Kingdom Atomic Energy Authority, Culham Centre for Fusion Energy, Culham Science Centre, Abingdon, Oxon OX14 3DB, United Kingdom

${ }^{3}$ Center for Energy Research (CER), University of California-San Diego (UCSD), La Jolla, California 92093, USA
\end{abstract}

a)Electronic mail: fabio.riva@ukaea.uk

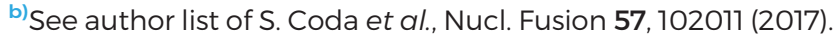

\begin{abstract}
The effect of plasma shaping on scrape-off layer (SOL) plasma turbulence is investigated through a rigorous validation exercise. Two- and three-dimensional simulations of the SOL plasma dynamics in three TCV limited discharges are carried out with the GBS code. These discharges realize an almost circular magnetic equilibrium, an elongated equilibrium, and an elongated equilibrium with negative triangularity. For the three plasma discharges, three simulations are performed, considering (i) a three-dimensional model with an explicit dependence on elongation, triangularity, and the inverse aspect ratio; (ii) a circular three-dimensional model in the infinite aspect ratio limit; and (iii) a twodimensional model, which assumes a circular magnetic equilibrium in the infinite aspect ratio limit, cold ions, and interchange driven turbulence in the sheath limited regime. Ten validation observables common to simulations and experimental measurements from a reciprocating probe located at the TCV outer midplane are identified, and the agreement between experimental and numerical results relative to each observable is evaluated. The composite metric introduced by Ricci et al. [Phys. Plasmas 18, 032109 (2011)] is then used to assess the overall agreement between simulations and experimental measurements. It is found that the shaping model implemented in GBS improves the description of SOL plasma turbulence, taking into account the impact of elongation and triangularity.
\end{abstract}

Published under license by AIP Publishing. https://doi.org/10.1063/1.5123451

\section{INTRODUCTION}

Since simulation codes are playing an increasingly important role in optimizing and predicting the performance of present and future fusion devices, there is increasing motivation in the fusion community in developing and applying verification and validation $(V \& V)$ procedures. ${ }^{1-3}$ Verification is "the process of determining that a model implementation accurately represents the developer's conceptual description of the model and the solution to the model," while validation is "the process of determining the degree to which a model is an accurate representation of the real world from the perspective of the intended uses of the model." ${ }^{4}$

In the past few years, great effort was devoted to the development of $\mathrm{V} \& \mathrm{~V}$ procedures for plasma turbulence simulations. Besides code to code comparison (also known as code benchmark, see, e.g., Refs. 5-10), rigorous verification methodologies based on convergence studies, such as the method of manufactured solutions, ${ }^{11}$ are now also routinely used (see, e.g., Refs. 12-16). Guidelines for performing a rigorous validation in the context of magnetic confinement fusion energy were laid down by the seminal works of Terry et al. ${ }^{17}$ and Greenwald. ${ }^{18}$ Main objectives of validation procedures include the quantification of the degree of agreement of the code results, and therefore of the physics model, with experimental data to assess the maturity of our understanding of fusion-relevant systems; the comparison between different models to determine the most suitable one for describing a physical system; and the identification of parameter regimes where improvements to the current available models are 
needed. An overview of best practices and examples of validation in fusion is given in Refs. 19-22. These examples reveal that the validation effort carried out by the fusion community has mostly focused on core turbulence.

The goal of the present paper, which is an extension of the work illustrated in Chap. 7 of Ref. 23, is twofold. First, we apply for the first time the validation methodology proposed in Ref. 24 to turbulence in tokamak scrape-off layer (SOL) conditions. More precisely, we simulate the turbulent SOL plasma dynamics in three limited discharges realized on the TCV tokamak ${ }^{25}$ with different equilibrium magnetic shapes. We then rigorously validate the numerical results against experimental measurements. Second, we assess the maturity of our understanding of plasma shaping effects on SOL turbulence, thus validating the shaping model recently introduced in GBS. ${ }^{26}$ As a matter of fact, there is experimental evidence that plasma shaping plays a strong role in regulating plasma turbulence, both in the core and in the SOL. ${ }^{27,28}$ We note that, while the properties of SOL plasma turbulence in TCV have been investigated in the past both experimentally and numerically (see, e.g., Refs. 29-33) and the physics mechanisms behind the effect of plasma shaping on turbulence are discussed in Ref. 26, the present work focuses on the application of the rigorous validation methodology to investigate the impact of plasma shaping on SOL plasma dynamics.

Because of the large collisionality typical of the tokamak SOL, plasma turbulence in this region is generally simulated by employing a set of drift-reduced Braginskii equations. ${ }^{34,35}$ These equations have been implemented in a number of simulation codes, both twodimensional and three-dimensional, that have been extensively used to investigate the plasma dynamics at the periphery of fusion devices (see, e.g., Refs. 14, 16, and 36-42) Herein, we use three different models, all based on the drift-reduced Braginskii equations, considering (i) a three-dimensional model of SOL plasma turbulence in noncircular magnetic geometry, where we use the analytical equilibrium model discussed in Ref. 26 to introduce the dependence of the magnetic field on tokamak inverse aspect ratio, Shafranov's shift, elongation, and triangularity; (ii) a three-dimensional model in circular magnetic geometry and in the infinite aspect ratio limit; and (iii) a two-dimensional model in circular geometry and in the cold ion and interchange driven turbulence limits. All these models are implemented in the GBS code. ${ }^{38,43}$ Three different simulations are carried out with each model, considering three TCV plasma discharges with (i) an almost circular magnetic equilibrium, (ii) plasma elongation $\kappa=1.53$, and (iii) plasma elongation $\kappa=1.57$ and triangularity $\delta=-0.19$. The simulation results are then compared with TCV experimental measurements obtained with a horizontal reciprocating probe located at the tokamak outer midplane. ${ }^{44}$ To carry out the validation, we consider the validation methodology introduced by Ricci et al. in Refs. 24 and 45, used for validating plasma turbulence simulations of the TORPEX device ${ }^{46}$ against experimental measurements. This methodology can be summarized as follows. First, a number of physical quantities to be compared common to the experimental measurements and simulation results, referred to as validation observables, are identified and organized into a hierarchy, which is based on the number of model assumptions and combinations of measurements necessary to obtain the observable. Then, the agreement between experimental measurements and the simulations is evaluated considering the experimental and numerical uncertainties relative to each observable. Combining the result of the comparison of all the observables, while taking into account their position in the hierarchy and the uncertainties affecting them, the overall agreement between simulations and experiments is quantified by using an appropriate composite metric, $\chi$. Finally, the metric $\chi$ is complemented by an index, $Q$, used to assess the quality of the comparison. A more detailed description of this methodology is presented in Sec. II. For the present study, ten observables are considered and the composite metric $\chi$ is used to quantify the level of agreement between the simulations and the experiments for the three models and assess the maturity of our understanding of plasma shaping effects on SOL turbulence.

The present paper is structured as follows. After this introduction, in Sec. II, we summarize the validation methodology used in this study. Then, in Sec. III, we discuss the three models considered for this investigation. In Sec. IV, we illustrate the TCV experimental setup, the probes used to collect the experimental measurements, and the simulations we carried out. We present the validation observables considered for our analysis and the agreement between experimental measurements and numerical results with respect to every single observable in Sec. V. Finally, the validation metric $\chi$ and the quality factor $Q$ are computed for the three models and discussed in Sec. VI.

\section{VALIDATION METHODOLOGY}

We briefly summarize the validation methodology introduced by Ricci et al. in Refs. 24 and 45. To carry out a validation exercise, a number of independent and relevant physical quantities, common to the experiment and the simulations, should be first identified and analyzed using the same technique. The values of the $j-$ th observable at points $i=1, \ldots, N_{j}$ (the index $i$ can be used to discretize time and/or multidimensional spatial coordinates), as coming from experimental measurements or simulation results, are denoted as $e_{i, j}$ and $s_{i, j}$, respectively. Second, the measure of the distance $d_{j}$ between experimental measurements and simulation results for the $j-$ th observable is computed as

$$
d_{j}=\sqrt{\frac{1}{N_{j}} \sum_{i=1}^{N_{j}} \frac{\left(e_{j, i}-s_{j, i}\right)^{2}}{\Delta e_{j, i}^{2}+\Delta s_{j, i}^{2}},}
$$

where $\Delta e_{j, i}$ and $\Delta s_{j, i}$ are the uncertainties affecting $e_{i, j}$ and $s_{i, j}$, respectively. We note that here we normalize the difference between $e_{j, i}$ and $s_{j, i}$ to the sum of the squares of their uncertainties, as we are interested in understanding if the basic physics mechanisms at play in the system are well captured by the model under consideration. The normalization to the actual value of the observable is instead preferable in the case that the predictive capabilities of the code are tested.

The level of agreement $R_{j}$ between simulations and experiments with respect to the observable $j$ is given by

$$
R_{j}=\frac{\tanh \left[\left(d_{j}-1 / d_{j}-d_{0}\right) / \lambda\right]+1}{2},
$$

such that $R_{j}=0$ denotes perfect agreement and $R_{j}=1$ denotes complete disagreement. Here, $d_{0}$ and $\lambda$ are parameters used to set the threshold level for agreement and sharpness of transition from agreement with disagreement, respectively. In the following, we consider $d_{0}=1$ and $\lambda=0.5$, as suggested in Ref. 45 . This choice implies that $R_{j} \approx 0.5$ when the discrepancy between the $j-$ th experimental and numerical observables is comparable to their uncertainties. The impact 
of the choice of these parameters on the results presented in this publication is discussed in Sec. VI.

Finally, the overall agreement between simulations and experiments is measured by introducing a composite metric

$$
\chi=\frac{\sum_{j} R_{j} H_{j} S_{j}}{\sum_{j} H_{j} S_{j}},
$$

which combines the level of agreement of each observable, $R_{j}$, with its weight, $H_{j} S_{j}$, which defines how stringent each observable is for comparison purposes, such that $\chi=0$ means perfect agreement and $\chi=1$ complete disagreement. Here, $H_{j}$ and $S_{j}$ denote the weight of each observable $j$ according to the number of assumptions made for its evaluation and the level of confidence characterizing its measurement, respectively. More precisely, $H_{j}$ accounts approximately for the uncertainties related to model assumptions and/or to combinations of measurements, which are often needed to estimate the validation observables from the simulation results and the raw experimental data, ${ }^{47}$ and are generally very challenging to evaluate. In practice, the observables are organized into a hierarchy based on the number of assumptions required for their evaluation, $h_{j}$. Since the higher the hierarchy level of an observable is, the lower its importance in the comparison metric should be, $H_{j}$ is a decreasing function of $h_{j}$. Following Ref. 24, we define $H_{j}=1 / h_{j}$. On the other hand, the quantity $S_{j}$ is used to assess the precision of the measurement of the $j-$ th observable and should be a decreasing function of the experimental and simulation uncertainties. Reference 24 proposes

$$
S_{j}=\exp \left(-\frac{\sum_{i} \Delta e_{j, i}+\sum_{i} \Delta s_{j, i}}{\sum_{i}\left|e_{j, i}\right|+\sum_{i}\left|s_{j, i}\right|}\right),
$$

i.e., $S_{j}=1$ if $\Delta e_{j, i}=0$ and $\Delta s_{j, i}=0$, while $S_{j}$ vanishes if very large uncertainties affect $e_{j}$ and $s_{j}$. Reference 24 also introduces the index

$$
Q=\sum_{j} H_{j} S_{j}
$$

used to assess the quality of the comparison itself. The idea is that a validation is more reliable with a large number of observables and if their weight $H_{j} S_{j}$ is large.

Finally, we note that the methodology we use here is mostly targeted to discriminate among models and assess whether they follow the experimental trends or not. On the other hand, it is much more delicate to judge a single model in absolute terms and establish if the observed level of agreement is acceptable or not, i.e., if the code is "validated" or not. Therefore, in the present publication, we focus on the $\chi$ dependence on the magnetic geometry and on the simulation model, rather than on its absolute value.

\section{SIMULATION MODELS}

Since the tokamak SOL is characterized by rather low temperatures and relatively high collisionality, a fluid model based on the Braginskii equations $^{34}$ is typically employed to describe the plasma dynamics in this region. Moreover, because SOL turbulence occurs on timescales much slower than the gyromotion and it is usually characterized by spatial variations that occur on scale lengths longer than the ion gyroradius, the drift approximation is often adopted. The set of equations to describe plasmas in such conditions, known as drift-reduced Braginskii equations, ${ }^{35}$ was implemented in the GBS code to simulate plasma turbulence at the tokamak periphery. ${ }^{38,43}$

GBS is a three-dimensional flux-driven, two-fluid, turbulence code that solves the drift-reduced Braginskii equations numerically by using finite differences for the spatial discretization and a standard fourth-order Runge-Kutta method for the time advance. Increasingly complex magnetic configurations were considered in developing GBS. Initially, the code was used to simulate linear devices such as LAPD (Large Plasma Device) ${ }^{48}$ and simple magnetized toroidal devices such as TORPEX. ${ }^{49}$ GBS was then extended to limited SOL tokamak circular geometries. ${ }^{38}$ More recently, the capabilities of simulating noncircular geometries and diverted configurations were also introduced. ${ }^{26,50}$

For our study, we consider the GBS model in the electrostatic limit and we employ the Boussinesq approximation in evaluating the divergence of the polarization current to simplify the vorticity equation. We note that, since density fluctuations are typically quite large in the SOL (i.e., of amplitude comparable to the time-averaged quantities), the Boussinesq approximation is hard to justify from a theoretical point of view. Nevertheless, a relatively weak effect on SOL turbulence was found in recent studies, as discussed in Refs. 51-54. The resulting system of equations is written as follows:

$$
\begin{gathered}
\frac{\partial n}{\partial t}=-\frac{1}{B}\{\phi, n\}+\frac{2}{e B}\left[C\left(p_{e}\right)-e n C(\phi)\right] \\
-\nabla \cdot\left(n v_{\| e} \mathbf{b}\right)+\mathcal{D}_{n}(n)+S_{n}, \\
\frac{\partial \omega}{\partial t}=-\frac{1}{B}\{\phi, \omega\}+\frac{2 B}{m_{i} n} C\left(p_{e}+p_{i}\right)-v_{\| i} \nabla_{\|} \omega+\frac{B^{2}}{m_{i} n} \nabla \cdot\left(j_{\|} \mathbf{b}\right) \\
+\frac{B}{3 m_{i} n} C\left(G_{i}\right)+\mathcal{D}_{\omega}(\omega), \\
\frac{\partial v_{\| e}}{\partial t}=-\frac{1}{B}\left\{\phi, v_{\| e}\right\}+\frac{1}{m_{e}}\left[e \nabla_{\|} \phi-\frac{\nabla_{\|} p_{e}}{n}-0.71 \nabla_{\|} T_{e}\right. \\
\left.+\frac{e j_{\|}}{\sigma_{\|}}-\frac{2}{3 n} \nabla_{\|} G_{e}\right]-v_{\| e} \nabla_{\|} v_{\| e}+\mathcal{D}_{v_{\|} e}\left(v_{\| e}\right), \\
\frac{\partial v_{\| i}}{\partial t}=-\frac{1}{B}\left\{\phi, v_{\| i}\right\}-v_{\| i} \nabla_{\|} v_{\| i}-\frac{1}{m_{i} n} \nabla_{\|}\left(p_{e}+p_{i}\right) \\
\left.\frac{\partial T_{e}}{\partial t}=-\frac{1}{3 m_{i} n}\left\{\phi, T_{\|}\right\}+\frac{4 T_{e}}{3 e B}\left[\frac{C\left(p_{e}\right)}{n}+\frac{5}{2} C\left(T_{e}\right)-e C(\phi)\right]-v_{v_{\|} i} \nabla_{\| i}\right), \\
+\frac{\partial T_{e} T_{e}+S_{T_{e}}}{3}\left[0.71 \frac{\nabla \cdot\left(j_{\|} \mathbf{b}\right)}{e n}-\nabla \cdot\left(v_{\| e} \mathbf{b}\right)\right]+\nabla_{\|}\left(\chi_{\| e} \nabla_{\|} T_{e}\right)+\mathcal{D}_{T_{e}}\left(T_{e}\right), \\
+\frac{2 T_{i}}{3}\left[\frac{1}{B}\left\{\phi, T_{i}\right\}+\frac{4 T_{i}}{3 e B}\left[\frac{C\left(p_{e}\right)}{n}-\frac{5}{2} C\left(T_{i}\right)-e C(\phi)\right.\right. \\
\left.\frac{\partial T_{i}}{\partial t}-\nabla \cdot\left(v_{\| i} \mathbf{b}\right)\right]+\nabla_{\|}\left(\chi_{\| i} \nabla_{\|} T_{i}\right)+\mathcal{D}_{T_{i}}\left(T_{i}\right),
\end{gathered}
$$

where $\omega=\nabla_{\perp}^{2} \phi+\nabla_{\perp}^{2} T_{i} / e$ is the plasma vorticity, $j_{\|}=e n\left(v_{\| i}-v_{\| e}\right)$ the parallel current, $p_{e}=n T_{e}$ and $p_{i}=n T_{i}$ the electron and ion plasma pressures, $\chi_{\| e}$ and $\chi_{\| i}$ the parallel electron and ion thermal conductivities, $B$ the norm of the magnetic field, $\mathbf{b}$ the unitary vector oriented in the direction of $\mathbf{B}$, and $\sigma_{\|}=1.96 e^{2} n \tau_{e} / m_{e}$ the Spitzer conductivity, with $\tau_{e}$ the electron collision time. The density and temperature 
sources, $S_{n}, S_{T_{e}}, S_{T_{i}}$, are used to mimic the particles and heat outflow from the core, assuming that the plasma particles, recycled at the walls, migrate to the core as neutral atoms, where they are then ionized (i.e., we neglect ionization sources in the SOL region). The gyroviscous contributions are included in the model through the $G_{i}=-\eta_{0 i}\left[2 \nabla_{\|} v_{\| i}\right.$ $\left.+C(\phi) / B+C\left(p_{i}\right) /(e n B)\right]$ and $G_{e}=-\eta_{0 e}\left[2 \nabla_{\|} v_{\| e}+C(\phi) / B-C\left(p_{e}\right) /\right.$ $(e n B)]$ terms, with $\eta_{i}$ and $\eta_{e}$ the gyroviscous coefficients. ${ }^{34}$ Moreover, small perpendicular diffusion terms of the form $\mathcal{D}_{A}(A)=D_{A} \nabla_{\perp}^{2} A$, with $A=n, \omega, \phi, v_{\| i}, v_{\| e}, T_{e}, T_{i}$, are introduced for numerical stability reasons. Here, the Poisson brackets are defined as $\{\phi, A\}=\mathbf{b} \cdot(\nabla \phi$ $\times \nabla A)$, the curvature operator as $C(A)=B / 2[\nabla \times(\mathbf{b} / B)] \cdot \nabla A$, the perpendicular Laplacian as $\nabla_{\perp}^{2} A=-\nabla \cdot[\mathbf{b} \times(\mathbf{b} \times \nabla A)]$, and the parallel gradient as $\nabla_{\|} A=\mathbf{b} \cdot \nabla A$. Equations (6)-(11) are completed by a set of boundary conditions describing the plasma dynamics at the magnetic presheath entrance, as detailed in Refs. 55 and 56.

The dependence on the magnetic field geometry enters in the model through the norm of the magnetic field $B$, the direction of the unit vector $\mathbf{b}$, and the differential operators $\{\phi, A\}, C(A), \nabla_{\|}(A)$, and $\nabla_{\perp}^{2}(A)$. A model for expressing these quantities in limited, noncircular magnetic geometries has been recently introduced in GBS. ${ }^{26}$ This model can be used to investigate the effect of plasma elongation, $\kappa$, and plasma triangularity, $\delta$, on SOL turbulence. The magnetic field depends also on the tokamak major radius, $R_{0}$, the inverse aspect ratio, $\epsilon=a / R_{0}$ ( $a$ is the tokamak minor radius), the safety factor at the magnetic axis, $q_{0}$, and at the last closed flux surface (LCFS), $q_{a}$.

In order to validate the shaping model in Ref. 26 against experimental measurements and gain a deeper insight into the SOL turbulence properties, in the following we consider: (i) Equations (6)-(11) with the magnetic equilibrium given in Ref. 26, referred to as "Shaping model," (ii) Equations (6)-(11) with a circular equilibrium in the infinite aspect ratio limit, $\epsilon=0$, and with negligible Shafranov's shift, referred to as "Circular model"; and (iii) Equations (6)-(11) with a circular equilibrium in the cold ion and $\epsilon=0$ limits, reduced to a two-dimensional model assuming interchange driven turbulence and approximating the ion and electron flows at the magnetic presheath entrance as $\Gamma_{\| i}$ $=n \sqrt{T_{e} / m_{i}} / 2$ and $\Gamma_{\| e}=n \sqrt{T_{e} / m_{i}} \exp \left(\Lambda-e \phi / T_{e}\right) / 2$, with $\Lambda \approx 3.2$ for deuterium plasmas, referred to as " $2 \mathrm{D}$ model." We note that, when considering a circular equilibrium in the infinite aspect ratio limit (i.e., we impose $\epsilon=0, \Delta(r)=0, \kappa=1$, and $\delta=0$ ), the expressions for $\{\phi, A\}, C(A)$, and $\nabla_{\perp}^{2}(A)$ reduce to $\{\phi, A\}=\partial_{y} \phi \partial_{r} A-\partial_{r} \phi \partial_{y} A$, $C(A)=\left(\sin \theta \partial_{r} A+\cos \theta \partial_{y} A\right) / R$, and $\nabla_{\perp}^{2}(A)=\partial_{r}^{2} A+\partial_{y}^{2} A$ as detailed in Ref. 57, where $r$ and $y=a \theta$ are the radial and poloidal coordinates, respectively, with $\theta$ the poloidal angle. In this limit, Eqs. (6)-(11) reduce to Eq. (1) of Ref. 56.

In the approximation of a radially and poloidally local magnetic geometry, the 2D model equations are written as follows:

$$
\begin{aligned}
\frac{\partial n}{\partial t}= & -\frac{1}{B_{0}}\{\phi, n\}+\frac{2}{e R B_{0}}\left(\frac{\partial p_{e}}{\partial y}-e n \frac{\partial \phi}{\partial y}\right) \\
& -\frac{\sigma n c_{s}}{R} \exp \left(\Lambda-\frac{e \phi}{T_{e}}\right)+\mathcal{D}_{n}(n)+S_{n} \\
\frac{\partial \omega}{\partial t}= & -\frac{1}{B_{0}}\{\phi, \omega\}+\frac{2 B_{0}}{m_{i} R n} \frac{\partial p_{e}}{\partial y}+\frac{\sigma c_{s} \omega_{c i}^{2} m_{i}}{e R} \\
& \times\left[1-\exp \left(\Lambda-\frac{e \phi}{T_{e}}\right)\right]+\mathcal{D}_{\omega}(\omega) \\
\frac{\partial T_{e}}{\partial t}=-\frac{1}{B_{0}}\{\phi, & \left.T_{e}\right\}+\frac{4 T_{e}}{3 e R B_{0}}\left(\frac{1}{n} \frac{\partial p_{e}}{\partial y}+\frac{5}{2} \frac{\partial T_{e}}{\partial y}-e \frac{\partial \phi}{\partial y}\right) \\
-\frac{2 \sigma T_{e} c_{s}}{3 R} & {\left[1.71 \exp \left(\Lambda-\frac{e \phi}{T_{e}}\right)-0.71\right]+\mathcal{D}_{T_{e}}\left(T_{e}\right)+S_{T_{e}} }
\end{aligned}
$$

where $c_{s}=\sqrt{T_{e} / m_{i}}$ is the sound speed, $B_{0}$ is the norm of the magnetic field at the outer midplane of the LCFS, $\omega_{c i}=e B_{0} / m_{i}$ is the ion cyclotron frequency, and $\sigma=R / L_{c} \approx 1 /\left(2 \pi q_{a}\right)$, with $L_{c} \approx 2 \pi q R$ the connection length. Note that the $2 \mathrm{D}$ model equations correspond to Eqs. (B6)-(B8) in Ref. 45 and that $y$ is a coordinate in the binormal direction close to $\theta=0$.

\section{EXPERIMENTAL AND SIMULATION SETUPS}

The TCV experiment is a tokamak with major and minor radii $R_{0}=0.88 \mathrm{~m}$ and $a=0.25 \mathrm{~m}$, respectively. ${ }^{25}$ By being equipped with sixteen independent shaping and positioning coils, TCV has unique capabilitities of exploring a wide range of magnetic geometries, including negative triangularities, second-order $\mathrm{X}$ points, and more exotic plasma shapes. ${ }^{58}$ For this reason, TCV is an ideal test bed for validating the plasma shaping model introduced in Ref. 26 against experimental measurements.

In the following, we consider the three TCV inner-wall limited deuterium plasma discharges \#54147, \#55391, and \#55394. The experimental parameters measured for the three discharges are summarized in Table I and the poloidal cross sections of their magnetic surfaces are shown in Fig. 1. The plasma has an almost circular magnetic equilibrium in the discharge \#55391, an elongated equilibrium in the discharge \#55394, and an elongated equilibrium with negative triangularity in the discharge \#54147. The thick blue solid lines, representing the LIUQE ${ }^{59}$ reconstruction of the LCFS, are fitted with the model discussed in Ref. 26 (red dashed lines), showing a good agreement of the fitting with the magnetic reconstruction. We note that the three discharges have similar safety factors, $q_{a}$, densities, $n_{a}$, and tem-

\begin{tabular}{|c|c|c|c|c|c|c|c|c|c|c|}
\hline Discharge & $R_{0}(\mathrm{~m})$ & $\epsilon$ & $q_{a}$ & $\kappa$ & $\delta$ & $B_{0}(\mathrm{~T})$ & $n_{a}\left(10^{18} \mathrm{~m}^{-3}\right)$ & $T_{e, a}(\mathrm{eV})$ & $\rho_{s 0}(\mathrm{~mm})$ & $\nu_{e}^{*}$ \\
\hline \#54147 & 0.875 & 0.34 & 3.3 & 1.57 & -0.19 & 1.44 & $6 \pm 2$ & $37 \pm 15$ & 0.61 & 4.9 \\
\hline \#55391 & 0.870 & 0.30 & 3.4 & 1.07 & 0.00 & 1.45 & $4 \pm 1$ & $36 \pm 16$ & 0.60 & 3.5 \\
\hline \#55394 & 0.865 & 0.30 & 3.2 & 1.53 & 0.00 & 1.46 & $5 \pm 1$ & $41 \pm 14$ & 0.63 & 3.2 \\
\hline
\end{tabular}
peratures, $T_{e, a}$, at the LCFS. This allows us to decouple the influence of plasma shaping on the SOL dynamics from other effects. ${ }^{26}$

TABLE I. Tokamak major radius, inverse aspect ratio, edge safety factor, plasma elongation and triangularity, toroidal magnetic field on axis, plasma density and electron temperature at the LCFS, sound Larmor radius, and electron collisionality, $\nu_{e}^{*} \approx \pi q_{a} R / \lambda_{e}^{m f p}$, with $\lambda_{e}^{m f p}$ the electron mean free path, for the three TCV plasma discharges $\# 54147$, \#55391, and \#55394. The magnetic equilibrium parameters are obtained by fitting the profiles shown in Fig. 1 with the shaping model in Ref. 26. 

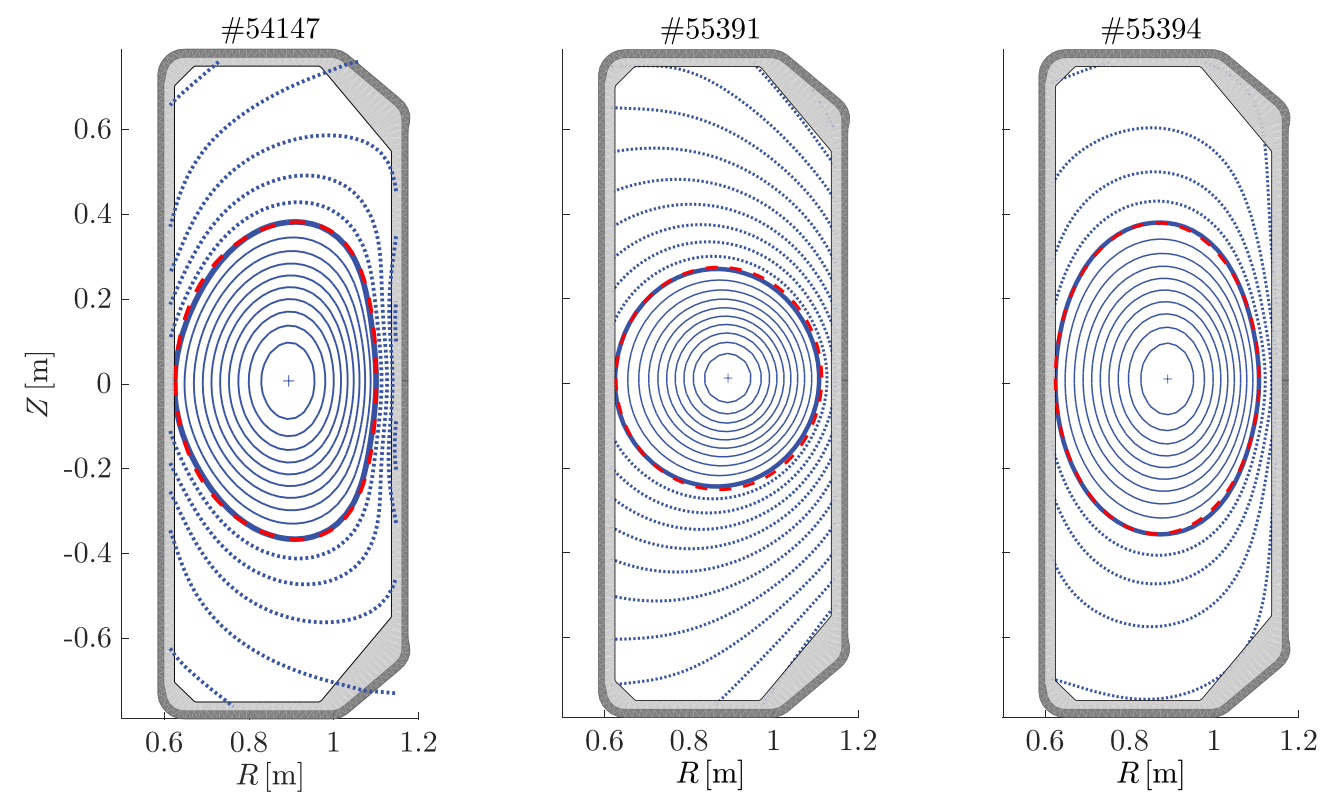

FIG. 1. Poloidal cross sections of the magnetic surfaces for the three TCV plasma discharges \#54147 (left panel), \#55391 (middle panel), and \#55394 (right panel). The blue lines indicate the LIUQE reconstruction, with thin continuous lines denoting the core flux surfaces, thick continuous lines denoting the LCFS, and dashed lines denoting the open field line region. The red dashed lines represent the reconstruction of the LCFS with the shaping model discussed in Ref. 26.

The experimental measurements discussed in the following are obtained with a horizontal reciprocating probe located at the tokamak outer midplane. The probe takes approximately $60 \mathrm{~ms}$ to traverse the SOL inward and outward. During this time-interval, the probe provides high spatial resolution measurements of time-averaged and fluctuating physical quantities (we note that global plasma parameters such as the toroidal magnetic field, the plasma current, and the averaged plasma density are almost constant, with variations smaller than $5 \%$, during these measurements). It is equipped with a probe head having ten electrostatic pins (two are used as a swept double probe and provide measurements of $I_{\text {sat }}, n$, and $T_{e}$, one is used to obtain direct measurements of $I_{\text {sat }}$, five are used as floating Langmuir probes to measure $V_{f l}$, and the last two are used to measure the poloidal Mach number), all acquiring data at $2.5 \mathrm{MHz}$. Taking the difference of two of the $V_{f l}$ pins, it is possible to estimate the poloidal electric field $E_{\theta}$. Since global plasma parameters remain approximately constant and are equal to the nominal values given in Table I during the probe reciprocation, the radial profiles related to the measured quantities are obtained by sampling the measured time traces in intervals of $1 \mathrm{~ms}$ and considering each subsignal as an independent measurement at constant radial location. For a more detailed description of the reciprocating probe, we refer to Refs. 44 and 60.

To assess the reliability of the models illustrated in Sec. III, we compare nonlinear GBS simulations based on the three TCV plasma discharges discussed above with experimental TCV measurements. More precisely, for each TCV plasma discharge we perform three simulations, one with the Shaping model, one with the Circular model, and one with the 2D model considering the parameters listed in Table I. However, since the Circular model and the 2D model are obtained assuming a circular magnetic geometry in an infinite aspect ratio, for these two models we carry out the simulations with $\epsilon=0, \kappa=1$, and $\delta=0$. For our simulations, we use perpendicular diffusion coefficients $D_{A} \approx 0.1 \mathrm{~m}^{2} \mathrm{~s}^{-1}$, with $A$ indicating all evolved fields. We note that the impact of perpendicular diffusion coefficients of similar amplitude on GBS simulation results was investigated in Ref. 57. It was observed that the simulation results are not affected significantly by these coefficients. Based on this finding, in the following we neglect the uncertainties affecting the numerical results due to the numerical diffusion terms. The particle and temperature sources are assumed poloidally and toroidally constant, with radial dependence $S_{n}(x), S_{T_{e}}(x), S_{T_{i}}(x)$ $\propto \exp \left[-(x-a+\sigma)^{2} / \sigma^{2}\right]$, where $\sigma=2.5 \rho_{s 0}$ and $\rho_{s 0}$ is the sound Larmor radius reported in Table I and $x$ is the radial coordinate, and we adjust the amplitude of the sources such that the simulated plasma densities and temperatures agree within uncertainties with the experimental measurements at the LCFS. We use $q_{0}=1$ for the Shaping model simulations. The radial domain extends from the inner radius $x_{i}=a-30 \rho_{s 0}$ to the outer radius $x_{o}=a+70 \rho_{s 0}$. Ad hoc boundary conditions are applied at $x_{i}$ and $x_{o}$, with Neumann's boundary conditions used for $n, v_{\| e}, v_{\| i}, T_{e}$, and $T_{i}$, and Dirichlet's boundary conditions for $\omega$ and $\phi$. To mitigate the impact of these boundary conditions on the simulation results, the two regions extending from $x=x_{i}$ to $x=a$, and from $x=a+2.5 \mathrm{~cm}$ to $x=x_{o}$ are considered as buffers and are not included in the analysis of the results. Moreover, for the three-dimensional simulations we consider an ion to electron temperature ratio $T_{e} / T_{i}=1$ at the LCFS, as indicated by typical charge exchange measurements in TCV. We note that the threedimensional simulations require three rather large numerical grids, i.e., $\left(N_{x}, N_{y}, N_{z}\right)=(128,1280,196),(128,1280,188),(128,1280,200)$, which correspond to a grid spacing $\Delta x \simeq 0.8 \rho_{s 0}, \Delta y \simeq 2 \rho_{s 0}$, and $\Delta \zeta=L_{\|} / 320$, with $z$ and $\zeta$ the toroidal and parallel coordinates, $L_{\|}$the parallel connection length from the outer midplane to the target, and $N_{x}, N_{y}$, and $N_{z}$ the number of grid points in $x, y$, and $z$, respectively. 
For this reason, we use a reduced ion to electron mass ratio, $m_{i} / m_{e}=800$, and a reduced parallel electron thermal conductivity, $\chi_{\| e} \approx 5 \times 10^{5} \mathrm{~m}^{2} \mathrm{~s}^{-1}$ (this corresponds to a reduction by approximately a factor $30-50$ with respect to the physical value). The value of these parameters decreases considerably the computational cost of our simulations. We note that the impact of a reduced electron parallel conductivity on the results of GBS simulations in a limited configuration was investigated in Ref. 26, showing that an increase in $\chi_{\| e}$ by a factor of 500 led to approximately a $20 \%$ steepening of the profiles. Additionally, we performed a linear investigation of the instabilities that govern the SOL turbulent transport with the linear model in Ref. 26, observing a reduction of $L_{p}$ by approximately $20 \%-25 \%$ when considering a realistic mass ratio with respect to $m_{i} / m_{e}=800$. Whereas the artificial values of $\chi_{\| e}$ and $m_{i} / m_{e}$ might represent an additional source of uncertainty on the numerical results, the detailed investigation of their impact on the validation observables is at the moment impractical. Therefore, in the following we neglect the uncertainties on the numerical results related to these two parameters. We rather focus on a sensitivity analysis in the value of the resistivity. This was shown to play an extremely important role in determining the turbulent regime in the SOL and its properties. ${ }^{61}$ Therefore, to investigate how experimental uncertainties on $n_{0}$ and $T_{e 0}$ impact the numerical results, we performed an additional Shaping model simulation of the plasma discharge \#54147 using $\sigma_{\|}$ten times smaller than its estimated Spitzer value. For the two-dimensional simulations, we use the numerical grid $\left(N_{x}, N_{y}\right)=(128,512)$, with the binormal direction extending over a distance $2 \pi a / q$.

\section{VALIDATION OBSERVABLES}

To validate the GBS simulations discussed in Sec. IV against TCV experimental measurements, we consider the following observables: the time-averaged plasma density, electron temperature, and ion saturation gradient lengths, $L_{n}, L_{T_{e}}$, and $L_{j_{s a t}}$; the root mean square (RMS) of $j_{\text {sat }}$ fluctuations normalized to the $j_{\text {sat }}$ time-averaged profile, $\delta j_{\text {sat }}^{R M S} / \bar{j}_{\text {sat }}$, the RMS profiles of floating potential and of electric field fluctuations, $\delta V_{f l}^{R M S}$ and $\delta E_{\theta}^{R M S}$; the $\delta j_{s a t}$ and $\delta V_{f l}$ probability distribution functions (PDF); and the $j_{\text {sat }}$ and $V_{f l}$ power spectralslopes, $\alpha_{j_{s a t}}$ and $\alpha_{V_{f}}$. Concerning the experimental measurements, we note that $n$ and $j_{\text {sat }}$ are obtained both from direct $I_{\text {sat }}$ and double probe measurements, $T_{e}$ is obtained by using the double probe only, $V_{f l}$ is obtained from one of the floating Langmuir probes, while $E_{\theta}$ is obtained by comparing the measurements of two poloidally separated floating potential Langmuir probes (i.e., we assume no difference in plasma temperature between the two electrodes). All measurements are taken twice, with the probe entering and exiting the plasma, providing at each radial position four independent measurements of $n$ and $j_{\text {sat }}$ and two independent measurements of all other plasma quantities.

Concerning the numerical results, these are obtained with simulations in turbulent quasisteady state on time intervals of approximately $200 \mu \mathrm{s}$, with an acquisition frequency of approximately $500 \mathrm{kHz}$. We note that $n$ and $T_{e}$ are direct output of GBS, $E_{\theta}$ is obtained by combining GBS time-traces of $\phi$ at two different poloidal locations, and we assume $j_{s a t} \propto n \sqrt{T_{e}+2 T_{i}}$, consistently with Ref. 62, and $V_{f l}=\phi-\left[\Lambda-\log \left(1+T_{i} / T_{e}\right) / 2\right] T_{e}$. However, since the 2D model does not evolve the ion temperature, we assume $T_{i}=T_{e}$ in evaluating $j_{s a t}$ and $V_{f l}$ from the two-dimensional results. In Secs. V A-V D we discuss in more detail the evaluation of all observables, focusing on their uncertainties, their hierarchy level, $h$, their level of agreement, $R$, and their accuracy parameter, $S$.

\section{A. Gradient scale lengths}

Some of the main uncertainties affecting the design and operation of future fusion devices are closely related to our ability of predicting the characteristic gradient lengths of SOL equilibrium profiles. Therefore, three of the validation observables considered in our study are the characteristic gradient lengths $L_{n}, L_{T_{e}}$, and $L_{j_{s a t}}$. For the plasma density, this is defined as $L_{n}=-\bar{n} / \partial_{x} \bar{n}$, where $\bar{n}(x)$ is the timeaveraged plasma density radial profile. Similar definitions apply to $L_{T_{e}}$ and $L_{j_{s a t}}$.

In TCV, the time-averaged radial profiles are evaluated by considering time-average windows of $1 \mathrm{~ms}$. In Figs. 2-4, we present the
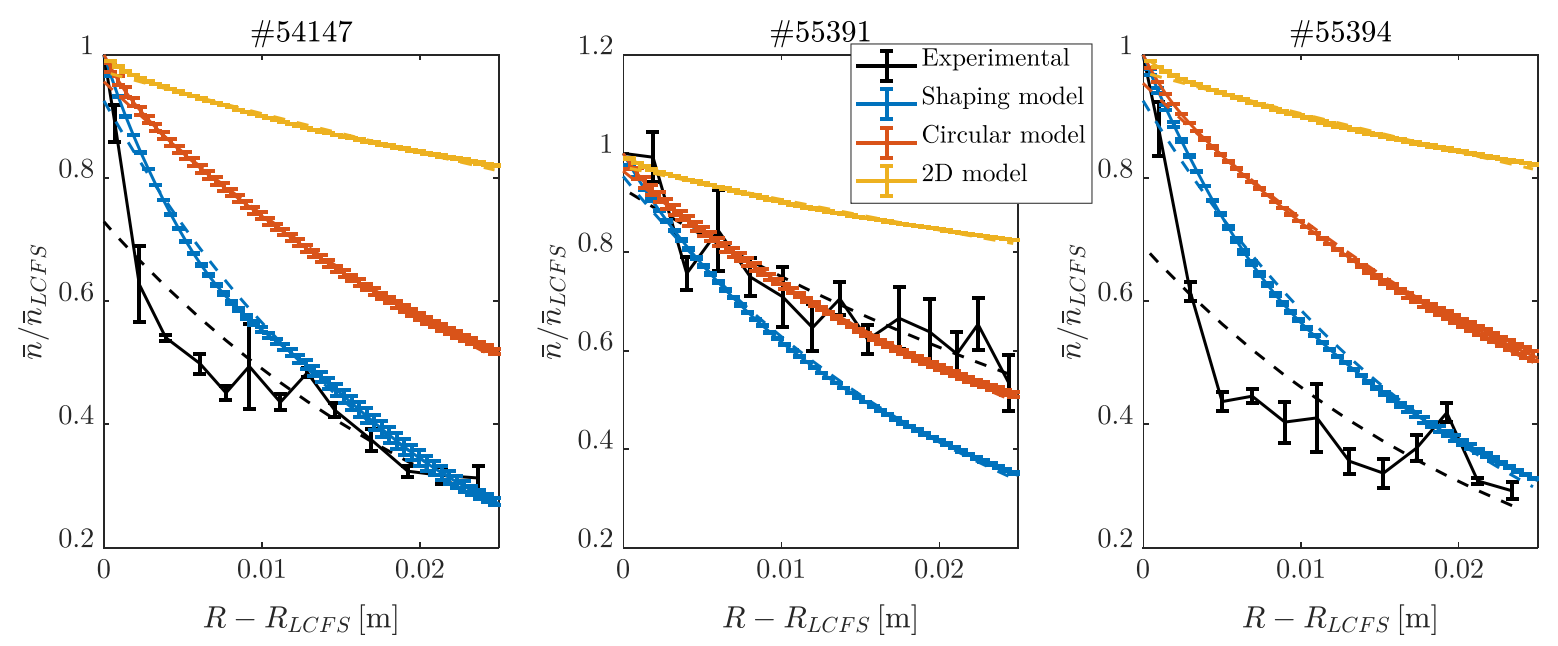

FIG. 2. Radial profiles of $\bar{n}$ normalized to $\bar{n}$ at the LCFS for the three plasma discharges \#54147, \#55391, and \#55394. The dashed lines denote an exponential fit between $x=0$ and $x=2.5 \mathrm{~cm}$. 

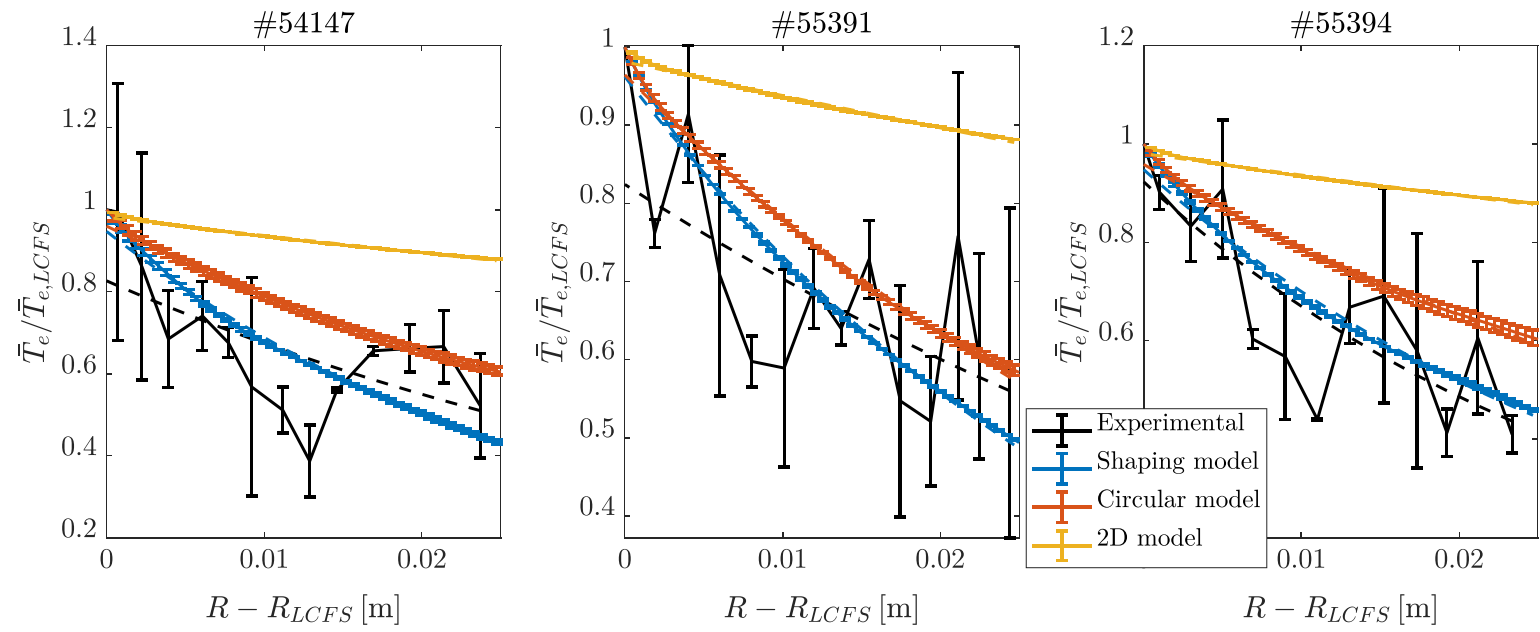

FIG. 3. Radial profiles of $\bar{T}_{e}$ normalized to $\bar{T}_{e}$ at the LCFS for the three plasma discharges \#54147, \#55391, and \#55394. The dashed lines denote an exponential fit between $x=0$ and $x=2.5 \mathrm{~cm}$

plasma density, electron temperature, and ion saturation current timeaveraged radial profiles. The $\bar{n}(x)$ profile is computed as the average over the four independent measurements and the uncertainties affecting it are estimated as $\Delta \bar{n}(x)=\operatorname{std}\left(\left|\bar{n}(x)-\bar{n}_{k}(x)\right|\right)$, with $k=1, \ldots, 4$ and $\bar{n}_{k}(x)$ the time-averaged plasma density resulting from the $k$-th independent measurement. Similar procedures are used to obtain $\bar{T}_{e}$ and $\bar{j}_{\text {sat }}$. Concerning the numerical results, the time-averaged profiles for the three-dimensional simulations are obtained by performing the toroidal- and time-averages of $n, T_{e}$, and $I_{\text {sat }}$ at the outer equatorial midplane. Similarly, for two-dimensional simulations the binormaland time-averaged profiles are considered. The resulting simulated radial profiles are shown in Figs. 2-4 (color lines), with their uncertainties estimated as the standard deviation of the time-averages carried out over two subintervals of approximately $100 \mu$ s. We observe that, for the Shaping model, elongation and negative triangularity result in steeper time-averaged profiles, in qualitative agreement with experimental measurements. Moreover, by comparing the threedimensional simulation results for the plasma discharge \#55391, we observe that Shafranov's shift and $\epsilon$ effects also result in steeper profiles, consistently with Ref. 26. Concerning the two-dimensional simulations, the profiles are considerably flatter in all cases, both if compared to experimental measurements and to three-dimensional simulation results.

The value of the characteristic gradient lengths is then obtained by fitting the radial profiles with an exponential. More specifically, for the experimental measurements we fit each of the four independent measurements assuming, e.g., $\bar{n}_{k}(x) \propto \exp \left(-x / L_{n, k}\right)$. We then compute $L_{n}$ as the average of the four resulting values. Similar procedures are used for $L_{T_{e}}$ and $L_{j_{s a t}}$. Figures 2-4 show that the experimental time-averaged radial profiles of $n, T_{e}$, and $j_{\text {sat }}$ are compatible with an
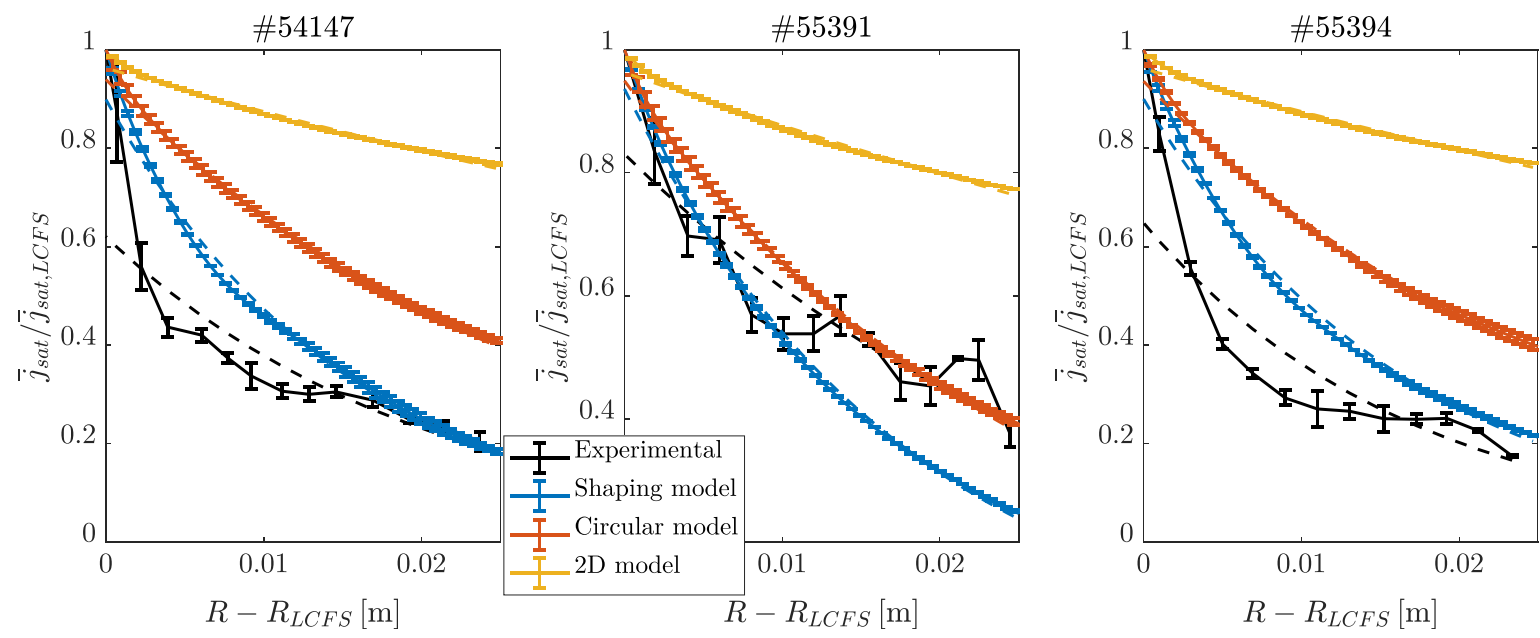

FIG. 4. Radial profiles of $\bar{j}_{\text {sat }}$ normalized to $\bar{j}_{\text {sat }}$ at the LCFS for the three plasma discharges \#54147, \#55391, and \#55394. The dashed lines denote an exponential fit between $x=0$ and $x=2.5 \mathrm{~cm}$. 
TABLE II. Plasma density, electron temperature and ion saturation current gradient scale lengths, and corresponding total uncertainties.

\begin{tabular}{|c|c|c|c|c|c|c|c|c|c|}
\hline & \multicolumn{3}{|c|}{ \#54147 } & \multicolumn{3}{|c|}{ \#55391 } & \multicolumn{3}{|c|}{ \#55394 } \\
\hline & $L_{n}(\mathrm{~cm})$ & $L_{T_{e}}(\mathrm{~cm})$ & $L_{j_{s a t}}(\mathrm{~cm})$ & $L_{n}(\mathrm{~cm})$ & $L_{T_{e}}(\mathrm{~cm})$ & $L_{j_{s a t}}(\mathrm{~cm})$ & $L_{n}(\mathrm{~cm})$ & $L_{T_{e}}(\mathrm{~cm})$ & $L_{j_{s a t}}(\mathrm{~cm})$ \\
\hline Experimental & $2.3 \pm 0.6$ & $6.3 \pm 12.6$ & $1.8 \pm 0.6$ & $5.3 \pm 2.4$ & $6.4 \pm 6.1$ & $3.4 \pm 1.1$ & $2.2 \pm 0.8$ & $3.4 \pm 2.5$ & $1.7 \pm 0.5$ \\
\hline Shaping model & $2.0 \pm 0.1$ & $3.1 \pm 0.2$ & $1.6 \pm 0.1$ & $2.4 \pm 0.1$ & $3.7 \pm 0.1$ & $1.8 \pm 0.1$ & $2.2 \pm 0.1$ & $3.1 \pm 0.1$ & $1.7 \pm 0.1$ \\
\hline Circular model & $3.9 \pm 0.1$ & $5.2 \pm 0.2$ & $2.9 \pm 0.1$ & $3.7 \pm 0.1$ & $4.8 \pm 0.1$ & $2.7 \pm 0.1$ & $3.8 \pm 0.2$ & $5.3 \pm 0.3$ & $2.8 \pm 0.1$ \\
\hline 2D model & $13.8 \pm 0.4$ & $21.7 \pm 0.6$ & $10.4 \pm 0.3$ & $14.2 \pm 0.5$ & $22.2 \pm 0.6$ & $10.7 \pm 0.3$ & $14.0 \pm 0.5$ & $22.0 \pm 0.7$ & $10.6 \pm 0.4$ \\
\hline
\end{tabular}

TABLE III. Level of agreement between simulations and experiments and accuracy parameters with respect to gradient scale lengths for the plasma discharge \#54147.

\begin{tabular}{lcccccc}
\hline \hline & $R_{L_{n}}$ & $S_{L_{n}}$ & $R_{L_{T_{e}}}$ & $S_{L_{T_{e}}}$ & $R_{L_{j_{s a t}}}$ & $S_{L_{j_{s a t}}}$ \\
\hline Shaping model & 0.00 & 0.85 & 0.00 & 0.26 & 0.00 & 0.83 \\
Circular model & 1.00 & 0.90 & 0.00 & 0.33 & 0.82 & 0.88 \\
2D model & 1.00 & 0.94 & 0.09 & 0.62 & 1.00 & 0.93 \\
\hline \hline
\end{tabular}

exponential decay (despite the fact that small deviations are observed for $\bar{n}$ and $\bar{j}_{\text {sat }}$ for the plasma discharges \#54147 and \#55391 and that, in general, decay lengths in the SOL are nonexponential, with a steeper profile near the LCFS and smaller gradients in the far $\mathrm{SOL}^{60,63,64}$ ). Similarly, Figs. 2-4 show that the radial profiles of $n, T_{e}$, and $j_{\text {sat }}$ obtained with GBS are compatible with an exponential decay (the only noticeable deviations are for $\bar{n}$ and $\bar{j}_{\text {sat }}$ with the Shaping model for the plasma discharges \#54147 and \#55394).

Concerning the experimental decay lengths, we consider two different sources of uncertainty. The first source is due to the best-fitting of the radial profiles with an exponential, referred to as $\Delta e_{L_{n}}^{f i t}$, while the second source is due to the uncertainties on the measurements of $\bar{n}_{k}(x)$, referred in the following as $\Delta e_{L_{n}}^{\text {meas }}$. For our validation, we take $\Delta e_{L_{n}}^{f i t}$ as given by the $95 \%$ confidence interval of the exponential fit of $\bar{n}(x)$ and we define $\Delta e_{L_{n}}^{\text {meas }}$ as the standard deviation of the four individual measurements. Finally, we obtain the total experimental uncertainty on $L_{n}$ as $\Delta e_{L_{n}}^{2}=\left(\Delta e_{L_{n}}^{\text {fit }}\right)^{2}+\left(\Delta e_{L_{n}}^{\text {meas }}\right)^{2}$ if $\Delta e_{L_{n}}^{\text {meas }}>\Delta e_{L_{n}}^{\text {fit }}$, while we consider $\Delta e_{L_{n}}=\Delta e_{L_{n}}^{\text {fit }}$ otherwise. The same procedure is used for evaluating the uncertainties on $L_{T_{e}}$ and $L_{j_{s a t}}$. The resulting experimental gradient scale lengths and the corresponding total uncertainties are reported in Table II. We see that elongation and negative triangularity reduce the experimental gradient scale lengths, consistently with the results reported in Ref. 26.

TABLE IV. Level of agreement between simulations and experiments and accuracy parameters with respect to gradient scale lengths for the plasma discharge \#55391.

\begin{tabular}{lcccccc}
\hline \hline & $R_{L_{n}}$ & $S_{L_{n}}$ & $R_{L_{T_{e}}}$ & $S_{L_{T_{e}}}$ & $R_{L_{\text {sat }}}$ & $S_{L_{\text {jsat }}}$ \\
\hline Shaping model & 0.09 & 0.73 & 0.00 & 0.54 & 0.31 & 0.81 \\
Circular model & 0.00 & 0.77 & 0.00 & 0.57 & 0.00 & 0.83 \\
2D model & 1.00 & 0.87 & 0.99 & 0.79 & 1.00 & 0.90 \\
\hline
\end{tabular}

Concerning the characteristic gradient lengths from the simulations, since the numerical results are evaluated on time intervals shorter than those considered for the experimental measurements, among the simulation uncertainties we also account for statistical fluctuations, $\Delta s_{L}^{\text {stat }}$. The values of $\Delta s_{L}^{\text {stat }}$ are obtained by averaging the simulation results on two subintervals of approximately $100 \mu \mathrm{s}$, computing $L$ for each subinterval, and evaluating $\Delta s_{L}^{\text {stat }}$ as the standard deviation of the two resulting gradient scale lengths. Finally, we use $\Delta s_{L}^{2}=\left(\Delta s_{L}^{\text {fit }}\right)^{2}+\left(\Delta s_{L}^{\text {stat }}\right)^{2}$ if $\Delta s_{L}^{\text {stat }}>\Delta s_{L}^{\text {fit }}$, while we consider $\Delta s_{L}=\Delta s_{L}^{\text {fit }}$ otherwise. We note that here we neglect numerical uncertainties and uncertainties due to input parameters on gradient scale lengths. The numerical uncertainties introduced by the time and space discretization are neglected because they are rather small, of the order of the grid spacing, for typical GBS simulations. ${ }^{12}$ The propagation of uncertainties on input parameters through model equations is neglected because they are typically smaller than $\Delta s_{L}^{\text {fit }}$ (for the threedimensional simulations this was assessed by performing a sensitivity scan in plasma resistivity, as discussed at the end of Sec. IV, while for the two-dimensional simulations this is discussed in detail in Ref. 65). The resulting gradient scale lengths and the corresponding total uncertainties are reported in Table II. We see that elongation and negative triangularity decrease the gradient scale lengths obtained with the Shaping model. This is in qualitative agreement with experimental measurements. Moreover, by comparing the three-dimensional simulation results for the plasma discharge \#55391, we observe that Shafranov's shift and $\epsilon$ effects reduce the SOL width, consistently with Ref. 26. For the two-dimensional simulations, we note that the 2D model overpredicts the gradient scale lengths in all cases, both if compared to experimental measurements and to three-dimensional simulation results. To carry out a more quantitative comparison, in Tables III-V we present the level of agreement between simulations and experiments with respect to the gradient scale lengths. Concerning the noncircular TCV discharges, we see that for the Shaping model $R_{L}$ is close to 0 for both discharges and for all quantities, while it is typically larger for the Circular model. This means that the Shaping model is generally in better agreement with the experimental measurements

TABLE V. Level of agreement between simulations and experiments and accuracy parameters with respect to gradient scale lengths for the plasma discharge \#55394.

\begin{tabular}{lcccccc}
\hline \hline & $R_{L_{n}}$ & $S_{L_{n}}$ & $R_{L_{T_{e}}}$ & $S_{L_{T_{e}}}$ & $R_{L_{j s a t}}$ & $S_{L_{\text {jat }}}$ \\
\hline Shaping model & 0.00 & 0.83 & 0.00 & 0.68 & 0.00 & 0.86 \\
Circular model & 0.90 & 0.86 & 0.00 & 0.72 & 0.98 & 0.87 \\
2D model & 1.00 & 0.93 & 1.00 & 0.88 & 1.00 & 0.94 \\
\hline
\end{tabular}


than the Circular model. On the other hand, the Circular model has slightly smaller $R_{L_{n}}$ and $R_{j_{s a t}}$ than the Shaping model for the \#55391 plasma discharge, showing for this discharge a slightly better agreement with the experimental measurements. This seems to suggest that Shafranov's shift and $\epsilon$ effects introduced in the Shaping model overstabilize the SOL plasma turbulence. In general, $R_{L} \simeq 1$ for the twodimensional simulations, meaning that they completely disagree from experimental measurements.

In Tables III-V, we also report the accuracy parameters for the gradient scale lengths. In most cases, uncertainties on gradient scale lengths are rather small and $S_{L}$ is typically larger than 0.7 . On the other hand, for the plasma discharges \#54147 and \#55391, large experimental uncertainties on the $\bar{T}_{e}$ measurements (as shown in Fig. 3) significantly reduce $S_{L_{T_{e}}}$. We also observe that, in general, a small $S_{L}$ corresponds to a small $R_{L}$. As a matter of fact, because of the normalization used in Eq. (1), large uncertainties affecting $e_{i, j}$ and $s_{i, j}$ result in a small $d_{j}$. However, we remind that quantities with large uncertainties will have a smaller weight in the combined metric $\chi$. Finally, since the only assumption made to obtain the gradient scale lengths from the $\bar{n}, \bar{T}_{e}$, and $\bar{j}_{\text {sat }}$ profiles is that the time-averaged radial profiles are exponentially decaying, and the uncertainties introduced by this assumption are already taken into account in $\Delta e_{L}$ and $\Delta s_{L}, h_{L}=h_{\bar{n}}$ $=h_{\bar{T}_{e}}=h_{\bar{j}_{\text {sat }}}=2$ as discussed in Ref. 47.

\section{B. Fluctuation amplitudes}

Important quantities used to characterize plasma turbulence in the tokamak SOL are related to the amplitude of the fluctuations. Therefore, in our validation we also consider the RMS $j_{\text {sat }}$ fluctuations normalized to the $j_{\text {sat }}$ background, $\delta j_{\text {sat }}^{R M S} / \bar{j}_{\text {sat }}$, the RMS floating potential fluctuations, $\delta V_{f l}^{R M S}$, and the RMS poloidal electric field fluctuations, $\delta E_{\theta}^{R M S}$.

Concerning the uncertainties affecting the experimental measurements, taking $\delta j_{\text {sat }}^{R M S} / \bar{j}_{\text {sat }}$ as an example, we compute $\left(\delta j_{\text {sat }}^{R M S} / \bar{j}_{\text {sat }}\right)$ for each independent measurements of $j_{\text {sat }}$. We then evaluate $\Delta e_{\delta j_{\text {sat }}^{\text {RaS }} / \bar{j}_{\text {sat }}}$ as the standard deviation of the four resulting values. The same procedure is used to evaluate $\Delta e_{\delta V_{f l}^{R M S}}$ and $\Delta e_{\delta E_{\theta}^{R M S}}$. We neglect other sources of uncertainty.

Concerning the numerical results, we consider three sources of uncertainty. The first one $\left(\Delta s_{\delta A}^{n u m}\right.$, with $\left.\delta A=\delta j_{\text {sat }}^{R M S}, \delta V_{f l}^{R M S}, \delta E_{\theta}^{R M S}\right)$ is introduced by discretizing the model equations in time and space, and it is evaluated by applying the solution verification procedure described in Ref. 12 to typical GBS simulations. We find that, for a resolution similar to the one used in this study, $\Delta s_{\delta j_{\text {sat }}^{n u m} / \bar{j}_{\text {sat }}}^{n u m} \approx 0.3 \delta j_{\text {sat }}^{R M S} /$ $\bar{j}_{s a t}, \Delta s_{\delta E_{\theta}^{R M S}}^{n u m} \approx 0.1 \delta E_{\theta}^{R M S}$, and $\Delta s_{\delta V_{f}^{R M S}}^{n u m} \approx 0.2 \delta V_{f l}^{R M S}$. The second source of uncertainty $\left(\Delta s_{\delta A}^{i n p}\right)$ is related to the propagation of uncertainties on input parameters. Since a tenfold increase in the plasma resistivity in the \#54147 simulation with the Shaping model leads to a $10 \%$ increase in SOL fluctuations, we assume $\Delta s_{\delta A}^{\text {inp }}=0.1 \delta A$. The last source of uncertainty $\left(\Delta s_{\delta A}^{s t a t}\right)$ is due to statistical fluctuations. This is evaluated by splitting the synthetic time traces into two subintervals, computing $\delta j_{\text {sat }}^{R M S} / \bar{j}_{\text {sat }}, \delta V_{f l}^{R M S}$, and $\delta E_{\theta}^{R M S}$ on each subinterval, and evaluating $\Delta s_{\delta A}^{\text {stat }}$ as the standard deviation of the resulting values. The total uncertainties are then computed as $\Delta s_{\delta A}^{2}=\left(\Delta s_{\delta A}^{\text {num }}\right)^{2}$ $+\left(\Delta s_{\delta A}^{i n p}\right)^{2}+\left(\Delta s_{\delta A}^{s t a t}\right)^{2}$.

The radial profiles of $\delta j_{\text {sat }}^{R M S} / \bar{j}_{\text {sat }}, \delta V_{f l}^{R M S}$, and $\delta E_{\theta}^{R M S}$ and the corresponding total uncertainties are displayed in Figs. 5, 6, and 7, respectively. We see that, in general, GBS simulations underestimate $j_{\text {sat }}$ fluctuations. This is consistent with previous observations, as discussed in Ref. 66. On the other hand, $E_{\theta}$ fluctuations are overestimated by all three models. In Fig. 6, we also observe that better agreement is found for $\delta V_{f l}^{R M S}$, in particular for the 2D model (we speculate that this a fortuitous event). To investigate more quantitatively the agreement between experimental measurements and simulation results with respect to the fluctuation amplitudes, in Tables VI-VIII we present $R_{\delta A}$ and $S_{\delta A}$. We see that, for all models, both $R_{\delta j_{s a t}^{R M S} / \bar{j}_{s a t}}$ and $R_{\delta E_{\theta}^{R M S}}$ are close to 1 , indicating that the simulation results and the experimental measurements typically disagree on the values of $\delta j_{\text {sat }}^{R M S} / \bar{j}_{\text {sat }}$ and $\delta E_{\theta}^{R M S}$. On the other hand, $R_{\delta V_{f}^{R M S}}$ is much smaller, indicating a better agreement for $\delta V_{f l}^{R M S}$, in particular for the $2 \mathrm{D}$ model. Concerning the
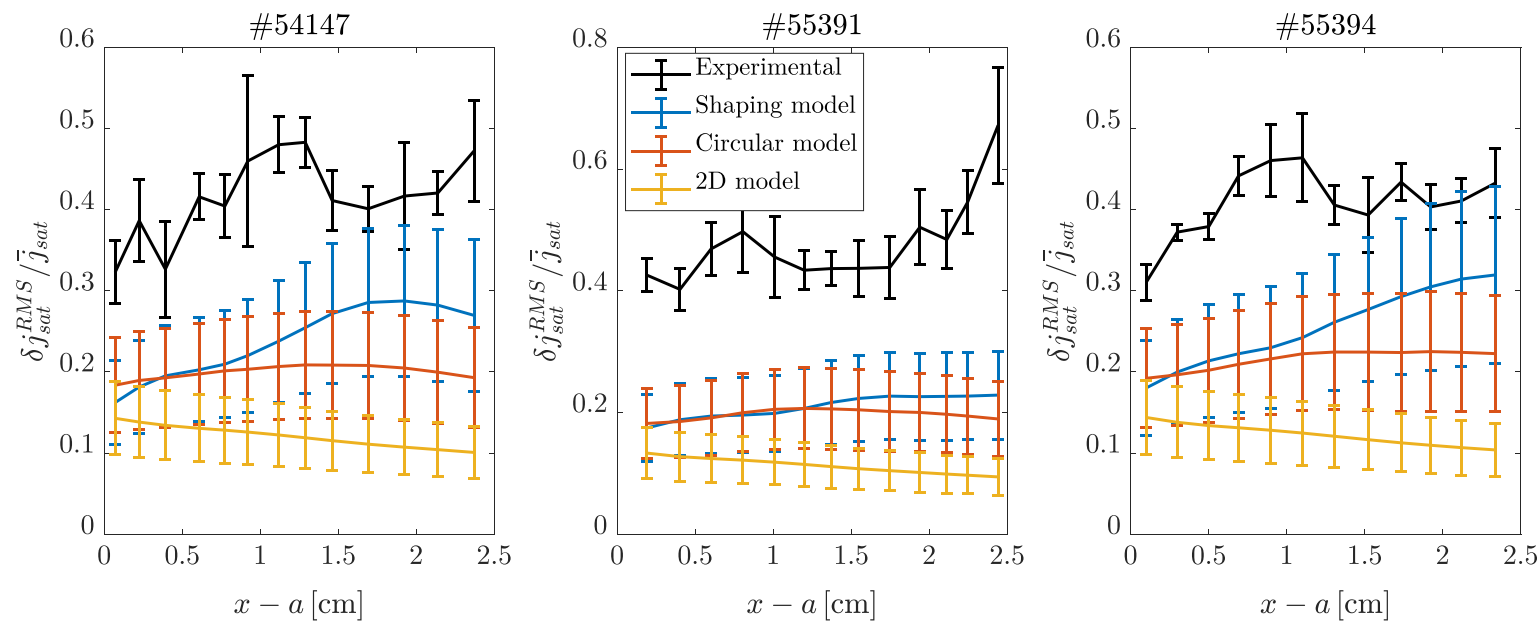

FIG. 5. Radial profiles of RMS $j_{\text {sat }}$ fluctuations normalized to the $j_{\text {sat }}$ background for the three plasma discharges $\# 54147$, \#55391, and \#55394. 

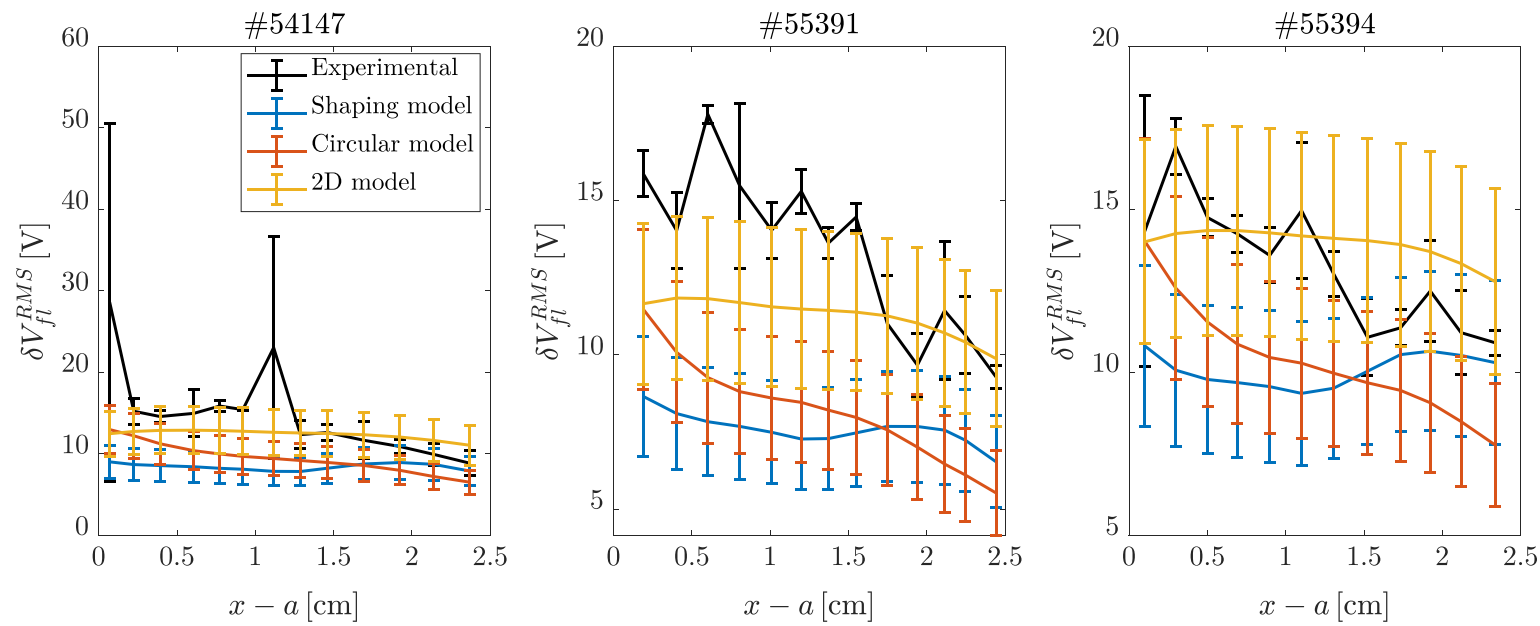

FIG. 6. Radial profiles of RMS $V_{f f}$ fluctuations for the three plasma discharges \#54147, \#55391, and \#55394.
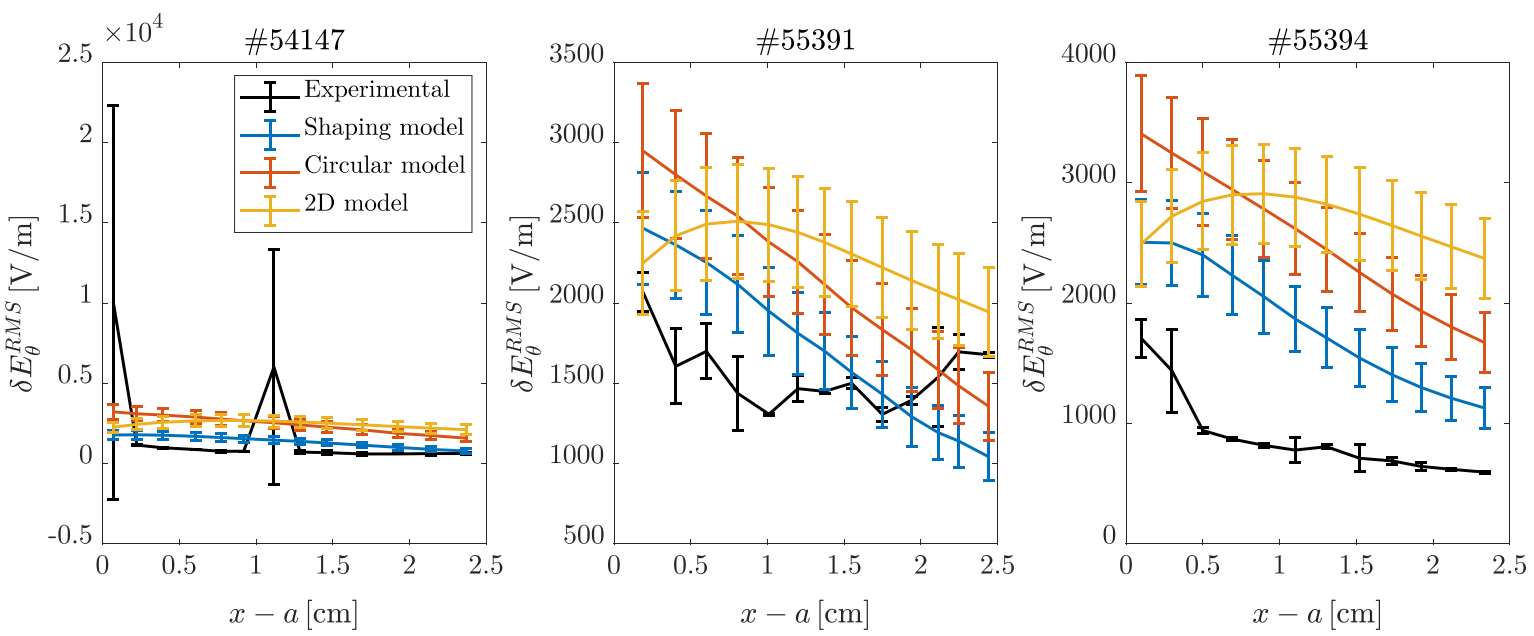

FIG. 7. Radial profiles of RMS $E_{\theta}$ fluctuations for the three plasma discharges \#54147, \#55391, and \#55394.

accuracy of the observables, we note that $S_{\delta A}>0.5$ for all models and plasma discharges, implying that the experimental and numerical uncertainties are rather small. Finally, as discussed in Ref. 47, we take $h_{\delta A}=2$.

\section{Probability distribution functions}

To gain a deeper insight into the properties of SOL plasma turbulence, for our validation we also consider the experimental and numerical PDFs of the $j_{\text {sat }}$ and $V_{f l}$ fluctuations at the three locations $x-a=0.5 \mathrm{~cm}, x-a=1.2 \mathrm{~cm}$, and $x-a=1.9 \mathrm{~cm}$, with the fluctuations normalized to their RMS value. This normalization is performed to avoid double counting the RMS values in the evaluation of $\chi$. The uncertainties affecting the experimental measurements are evaluated as the standard deviation between the two $\delta j_{s a t}$ and $\delta V_{f l}$ PDFs resulting from the probe entering and exiting the plasma. Concerning the numerical results, the numerical error affecting the $j_{\text {sat }}$ and $V_{f l}$
PDFs is estimated by considering the three nonlinear simulations discussed in Ref. 12, computing the $\delta j_{s a t}$ and $\delta V_{f l}$ PDFs at different radial locations, and performing a grid convergence analysis of the results. It is found that the maximum absolute numerical error is smaller than 0.02 . The statistical error affecting the three-dimensional simulation results has a similar amplitude, while for the two-dimensional

TABLE VI. Level of agreement between simulations and experiments and accuracy parameters with respect to RMS fluctuations for the plasma discharge \#54147.

\begin{tabular}{lcccccc}
\hline \hline & $R_{\delta j_{\text {sat }}^{R M S} / \bar{j}_{\text {sat }}}$ & $S_{\delta j_{\text {sat }}^{R M S} / \bar{j}_{\text {sat }}}$ & $R_{\delta V_{f l}^{R M S}}$ & $S_{\delta V_{f l}^{R M S}}$ & $R_{\delta E_{\theta}^{R M S}}$ & $S_{\delta E_{\theta}^{R M S}}$ \\
\hline Shaping model & 0.94 & 0.83 & 0.95 & 0.78 & 0.99 & 0.58 \\
Circular model & 1.00 & 0.84 & 0.34 & 0.78 & 1.00 & 0.65 \\
2D model & 1.00 & 0.85 & 0.00 & 0.78 & 1.00 & 0.65 \\
\hline \hline
\end{tabular}


TABLE VII. Level of agreement between simulations and experiments and accuracy parameters with respect to RMS fluctuations for the plasma discharge \#55391.

\begin{tabular}{lcccccc}
\hline \hline & $R_{\delta j_{\text {sat }}^{R M S} / j_{\text {sat }}}$ & $S_{\delta \delta_{\text {sat }}^{R M S} / j_{\text {sat }}}$ & $R_{\delta V_{f l}^{R M S}}$ & $S_{\delta V_{f l}^{R M S}}$ & $R_{\delta E_{\theta}^{R M S}}$ & $S_{\delta E_{\theta}^{R M S}}$ \\
\hline Shaping model & 1.00 & 0.84 & 1.00 & 0.87 & 0.81 & 0.90 \\
Circular model & 1.00 & 0.85 & 0.99 & 0.87 & 0.89 & 0.89 \\
2D model & 1.00 & 0.86 & 0.03 & 0.86 & 0.96 & 0.89 \\
\hline
\end{tabular}

TABLE VIII. Level of agreement between simulations and experiments and accuracy parameters with respect to RMS fluctuations for the plasma discharge \#55394.

\begin{tabular}{lcccccc}
\hline \hline & $R_{\delta j_{\text {sat }}^{R M S} / j_{\text {sat }}}$ & $S_{\delta j_{\text {sat }}^{R M S} / j_{\text {sat }}}$ & $R_{\delta V_{f l}^{R M S}}$ & $S_{\delta V_{f l}^{R M S}}$ & $R_{\delta E_{\theta}^{R M S}}$ & $S_{\delta E_{\theta}^{R M S}}$ \\
\hline Shaping model & 0.85 & 0.84 & 0.29 & 0.86 & 1.00 & 0.88 \\
Circular model & 0.99 & 0.85 & 0.08 & 0.86 & 1.00 & 0.88 \\
2D model & 1.00 & 0.88 & 0.00 & 0.85 & 1.00 & 0.88 \\
\hline \hline
\end{tabular}

simulations it is negligible. Therefore, in the following, we consider the conservative values $\Delta s_{P D F\left(\delta j_{s a t}\right)}=\Delta s_{P D F\left(\delta V_{f}\right)}=0.02$ and $\Delta s_{P D F(}\left(\delta j_{s a t}\right)$ $=\Delta s_{P D F\left(\delta V_{f}\right)}=0.04$ for the two- and three-dimensional simulation results, respectively.

The resulting PDFs and the corresponding uncertainties are displayed in Figs. 8 and 9. We observe that, despite some small differences, in particular near the center of the distributions, the simulation results and the experimental measurements are in quite good agreement.
The agreement between simulations and experiment is confirmed by computing the values of $R$, as shown in Tables IX and X. Indeed, at least for all three-dimensional simulations, $R<0.2$. In Tables IX and X we also report the parameter $S$. We see that $0.79<S<0.87$, indicating that the uncertainties affecting the PDFs are small. Finally, as discussed in Ref. 47, we take $h=2$ for the $\delta j_{s a t}$ and $\delta V_{f l}$ PDFs.

\section{Power spectral-slopes}

The last two observables considered in our comparison are the $j_{s a t}$ and $V_{f l}$ power spectral-slopes, $\alpha_{j_{s a t}}$ and $\alpha_{V_{f}}$. These are obtained by fitting the $j_{\text {sat }}$ and $V_{f l}$ power spectral densities (PSD) between $12 \mathrm{kHz}$ and $200 \mathrm{kHz}$ with a power function. More specifically, taking $j_{\text {sat }}$ as an example, we compute the $j_{\text {sat }}$ PSD at the three locations $x-a=0.5 \mathrm{~cm}, x-a=1.2 \mathrm{~cm}$, and $x-a=1.9 \mathrm{~cm}$. The results are then best-fitted between $f=12 \mathrm{kHz}$ and $f=200 \mathrm{kHz}$ assuming $\operatorname{PSD}_{j_{s a t}}(f) \propto f^{\alpha_{\text {sat }}}$. The same procedure is used to obtain $\alpha_{V_{f}}$.

Concerning the uncertainties affecting the experimental measurements, we consider the standard deviation between the two $\alpha_{j_{s a t}}$ and $\alpha_{V_{f}}$ resulting from the probe entering and exiting the plasma, $\Delta e^{\text {meas }}$. In addition, we consider the uncertainty related to best-fitting the $j_{\text {sat }}$ and $V_{f l}$ PSDs, $\Delta e^{f i t}$. This is evaluated as given by the $95 \%$ confidence interval of the best-fit of the PSDs. Finally, we assume $\Delta e^{2}$ $=\left(\Delta e^{f i t}\right)^{2}+\left(\Delta e^{\text {meas }}\right)^{2}$ if $\Delta e^{\text {meas }}>\Delta e^{\text {fit }}$, while we consider $\Delta e=\Delta e^{\text {fit }}$ otherwise.

Concerning the simulation results, we consider both the uncertainties related to best-fitting the PSDs with the power function, $\Delta s^{\text {fit }}$, and to the finite time-statistics, $\Delta s^{\text {stat }}$. The former are evaluated as discussed for the experimental PSDs. The latter are obtained by splitting
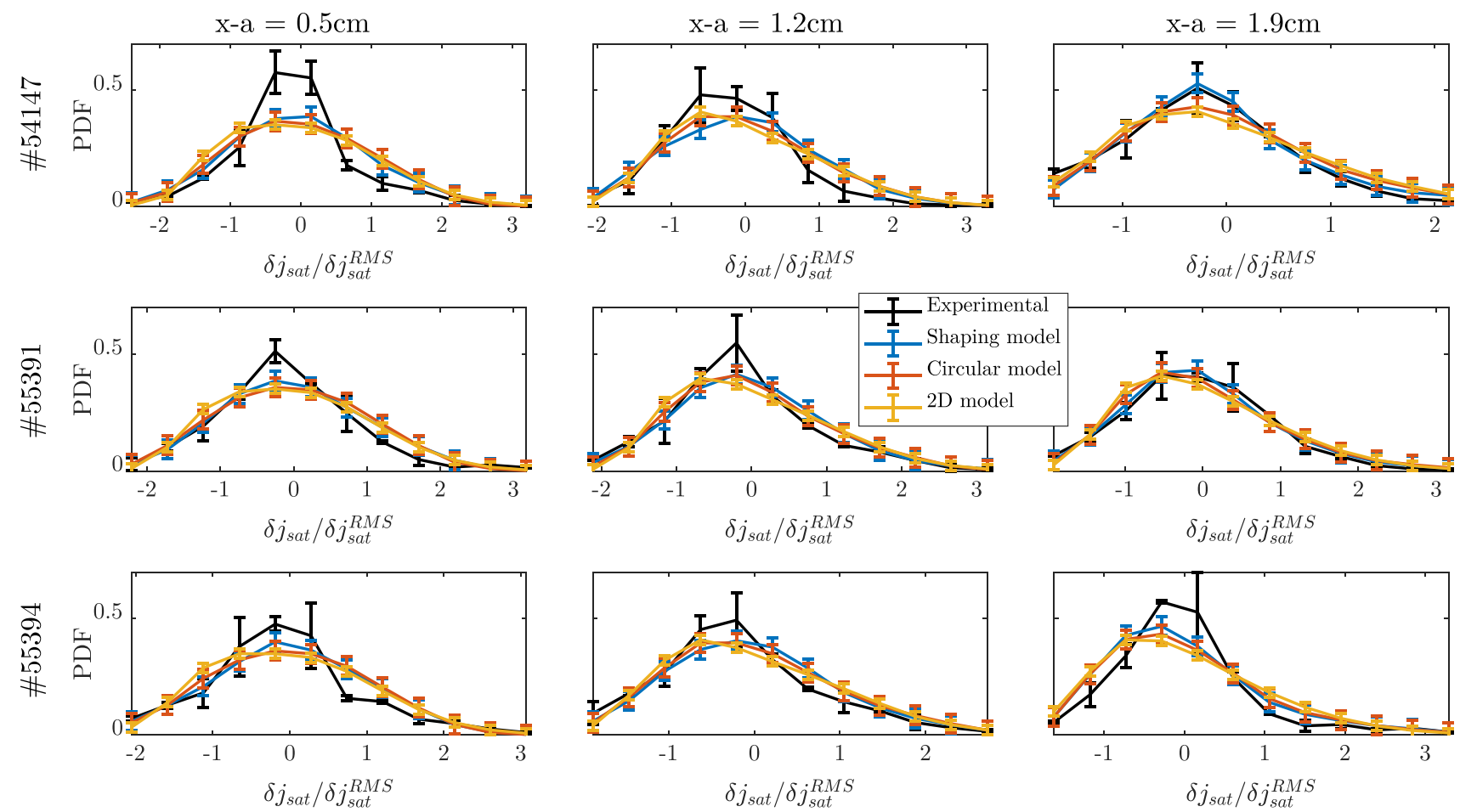

FIG. 8. PDF of $\delta j_{\text {sat }}$ at the three radial positions $x-a=0.5 \mathrm{~cm}, x-a=1.2 \mathrm{~cm}$, and $x-a=1.9 \mathrm{~cm}$ for the three plasma discharges \#54147, \#55391, and \#55394. 

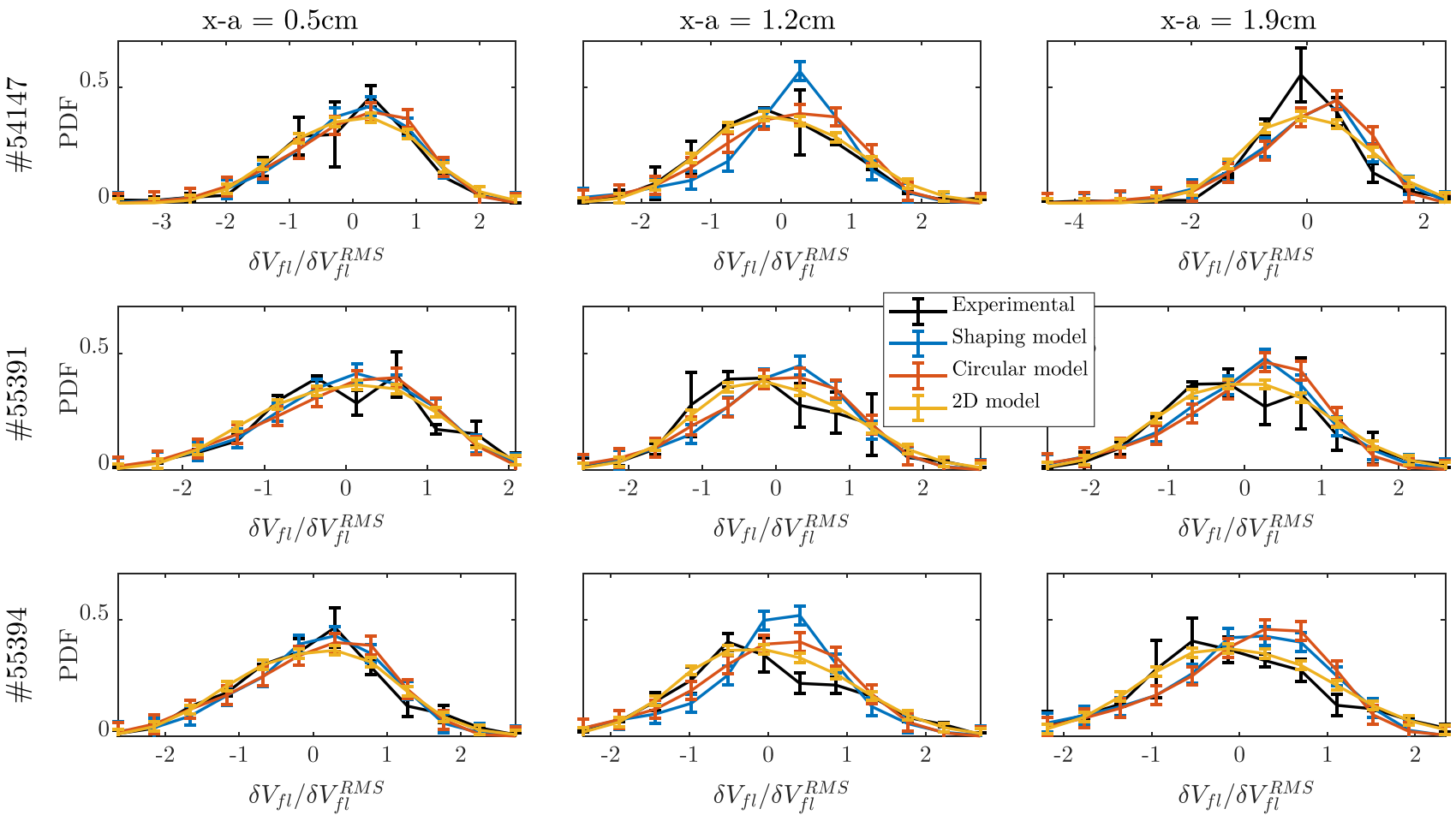

FIG. 9. PDF of $\delta V_{f l}$ at the three radial positions $x-a=0.5 \mathrm{~cm}, x-a=1.2 \mathrm{~cm}$, and $x-a=1.9 \mathrm{~cm}$ for the three plasma discharges $\# 54147$, \#55391, and \#55394.

TABLE IX. Level of agreement between simulations and experiments and accuracy parameter with respect to the $\delta j_{\text {sat }}$ PDFs.

\begin{tabular}{lcccccc}
\hline \hline & \multicolumn{2}{c}{$\# 54147$} & \multicolumn{2}{c}{$\# 55391$} & \multicolumn{2}{c}{$\# 55394$} \\
\cline { 2 - 7 } & $R_{\operatorname{PDF}\left(\delta j_{s a t}\right)} S_{\operatorname{PDF}\left(\delta j_{s a t}\right)} R_{\operatorname{PDF}\left(\delta j_{s a t}\right)} S_{\operatorname{PDF}\left(\delta j_{s a t}\right)} R_{\operatorname{PDF}\left(\delta j_{s a t}\right)} S_{\operatorname{PDF}\left(\delta j_{s a t}\right)}$ \\
\hline Shaping model & 0.01 & 0.82 & 0.00 & 0.82 & 0.03 & 0.82 \\
Circular model & 0.03 & 0.82 & 0.00 & 0.82 & 0.06 & 0.82 \\
2D model & 0.57 & 0.86 & 0.17 & 0.87 & 0.95 & 0.86 \\
\hline \hline
\end{tabular}

the time traces in two subintervals, computing $\alpha_{j_{s a t}}$ and $\alpha_{V_{f l}}$ on each subinterval, and evaluating $\Delta s^{f i t}$ as the standard deviation of the two resulting values. We note that numerical and input uncertainties are neglected here since for typical GBS simulations they are smaller than

TABLE X. Level of agreement between simulations and experiments and accuracy parameter with respect to the $\delta V_{f l}$ PDFs.

\begin{tabular}{lcccccc}
\hline \hline & \multicolumn{2}{c}{$\# 54147$} & \multicolumn{2}{c}{$\# 55391$} & \multicolumn{2}{c}{$\# 55394$} \\
\cline { 2 - 7 } & $R_{\operatorname{PDF}\left(\delta V_{f l}\right)} S_{\mathrm{PDF}\left(\delta V_{f l}\right)} R_{\operatorname{PDF}\left(\delta V_{f l}\right)} S_{\mathrm{PDF}\left(\delta V_{f l}\right)} R_{\operatorname{PDF}\left(\delta V_{f l}\right)} S_{\operatorname{PDF}\left(\delta V_{f f}\right)}$ \\
\hline Shaping model & 0.03 & 0.79 & 0.02 & 0.79 & 0.18 & 0.82 \\
Circular model & 0.02 & 0.79 & 0.04 & 0.79 & 0.08 & 0.82 \\
2D model & 0.03 & 0.85 & 0.01 & 0.84 & 0.00 & 0.86 \\
\hline \hline
\end{tabular}

$\Delta s^{f i t}$. The resulting power spectral-slopes and the corresponding total uncertainties are shown in Tables XI-XIII. We observe that the threedimensional models overestimate the power spectral-slopes, while agreement within uncertainties is found for the $\alpha_{V_{f}}$ obtained with the $2 \mathrm{D}$ model. This is confirmed by computing the level of agreement, as presented in Tables XIV and XV. Indeed, $R_{\alpha_{V_{f}}}<0.5$ for the $2 \mathrm{D}$ model. In Tables XIV and XV, we also present the parameters $S$. We see that $S>0.8$, indicating that the uncertainties affecting the power spectral-slopes are small.

Finally, as discussed in Ref. $47, h=2$ for the $j_{s a t}$ and $V_{f l}$ PSDs. Since the only assumption made to evaluate the power spectral-slopes from $I_{\text {sat }}$ and $V_{f l}$ measurements is that the PSDs are a power function of the frequency, and the uncertainty resulting from this assumption is taken into account in $\Delta e$ and $\Delta s$, we use $h=2$ also for $\alpha_{j_{s a t}}$ and $\alpha_{V_{f}}$.

\section{RESULTS AND DISCUSSION}

We now assess quantitatively the agreement between the three models presented in Sec. III and TCV experimental measurements by computing the metric $\chi$ and the quality factor $Q$. This combines the levels of agreement $R$, the hierarchy levels $h$, and the accuracy parameters $S$ illustrated in Sec. V. The results are presented in Table XVI.

Concerning the Shaping model, we observe that $\chi \simeq 0.45-0.48$ for all the considered TCV discharges, showing that this model is in similar overall agreement with experimental measurements for both circular and noncircular tokamak plasma discharges. On the other hand, $\chi$ increases above 0.6 by assuming a circular magnetic geometry in the infinite aspect ratio limit for the two negative triangularity and elongated plasma discharges \#54147 and \#55394. This is understood 
TABLE XI. Ion saturation current and floating potential spectral-slopes and corresponding total uncertainties at $x-a=0.5 \mathrm{~cm}$.

\begin{tabular}{|c|c|c|c|c|c|c|}
\hline & \multicolumn{2}{|c|}{ \#54147 } & \multicolumn{2}{|c|}{ \#55391 } & \multicolumn{2}{|c|}{ \#55394 } \\
\hline & $\alpha_{j_{s a t}}$ & $\alpha_{V_{f l}}$ & $\alpha_{j_{s a t}}$ & $\alpha_{V_{f l}}$ & $\alpha_{j_{s a t}}$ & $\alpha_{V_{f l}}$ \\
\hline Experimental & $-2.5 \pm 0.4$ & $-2.4 \pm 0.5$ & $-1.7 \pm 0.4$ & $-1.9 \pm 0.4$ & $-1.8 \pm 0.4$ & $-2.2 \pm 0.4$ \\
\hline Shaping model & $-1.6 \pm 0.1$ & $-1.3 \pm 0.1$ & $-1.25 \pm 0.05$ & $-1.2 \pm 0.1$ & $-1.4 \pm 0.1$ & $-1.2 \pm 0.1$ \\
\hline Circular model & $-1.20 \pm 0.04$ & $-1.3 \pm 0.1$ & $-1.3 \pm 0.1$ & $-1.3 \pm 0.1$ & $-1.2 \pm 0.1$ & $-1.3 \pm 0.1$ \\
\hline 2D model & $1.16 \pm 0.01$ & $-1.74 \pm 0.01$ & $-1.20 \pm 0.02$ & $-1.76 \pm 0.03$ & $-1.19 \pm 0.01$ & $-1.76 \pm 0.01$ \\
\hline
\end{tabular}

TABLE XII. Ion saturation current and floating potential spectral-slopes and corresponding total uncertainties at $x-a=1.2 \mathrm{~cm}$.

\begin{tabular}{|c|c|c|c|c|c|c|}
\hline & \multicolumn{2}{|c|}{ \#54147 } & \multicolumn{2}{|c|}{ \#55391 } & \multicolumn{2}{|c|}{ \#55394 } \\
\hline & $\alpha_{j_{s a t}}$ & $\alpha_{V_{f l}}$ & $\alpha_{j_{s a t}}$ & $\alpha_{V_{f l}}$ & $\alpha_{j_{s a t}}$ & $\alpha_{V_{f l}}$ \\
\hline Experimental & $-2.3 \pm 0.3$ & $-2.3 \pm 0.6$ & $-1.8 \pm 0.4$ & $-2.4 \pm 0.3$ & $-2.7 \pm 0.4$ & $-2.7 \pm 0.4$ \\
\hline Shaping model & $-1.5 \pm 0.1$ & $-1.4 \pm 0.1$ & $-1.1 \pm 0.1$ & $-1.1 \pm 0.1$ & $-1.3 \pm 0.1$ & $-1.2 \pm 0.1$ \\
\hline Circular model & $-1.2 \pm 0.1$ & $-1.2 \pm 0.1$ & $-1.3 \pm 0.1$ & $-1.2 \pm 0.1$ & $-1.2 \pm 0.1$ & $-1.2 \pm 0.1$ \\
\hline 2D model & $-1.03 \pm 0.01$ & $-1.77 \pm 0.02$ & $-1.07 \pm 0.02$ & $-1.84 \pm 0.02$ & $-1.06 \pm 0.01$ & $-1.82 \pm 0.01$ \\
\hline
\end{tabular}

TABLE XIII. Ion saturation current and floating potential spectral-slopes and corresponding total uncertainties at $x-a=1.9 \mathrm{~cm}$.

\begin{tabular}{|c|c|c|c|c|c|c|}
\hline & \multicolumn{2}{|c|}{ \#54147 } & \multicolumn{2}{|c|}{ \#55391 } & \multicolumn{2}{|c|}{ \#55394 } \\
\hline & $\alpha_{j_{s a t}}$ & $\alpha_{V_{f l}}$ & $\alpha_{j_{s a t}}$ & $\alpha_{V_{f l}}$ & $\alpha_{j_{s a t}}$ & $\alpha_{V_{f l}}$ \\
\hline Experimental & $-2.2 \pm 0.4$ & $-1.4 \pm 1.0$ & $-1.8 \pm 0.4$ & $-2.6 \pm 0.6$ & $-2.6 \pm 0.7$ & $-2.6 \pm 0.4$ \\
\hline Shaping model & $-1.8 \pm 0.1$ & $-1.9 \pm 0.1$ & $-1.33 \pm 0.05$ & $-1.5 \pm 0.1$ & $-1.5 \pm 0.1$ & $-1.7 \pm 0.1$ \\
\hline Circular model & $-1.4 \pm 0.1$ & $-1.6 \pm 0.1$ & $-1.5 \pm 0.1$ & $-1.6 \pm 0.1$ & $-1.5 \pm 0.1$ & $-1.6 \pm 0.1$ \\
\hline 2D model & $-1.19 \pm 0.02$ & $-2.03 \pm 0.02$ & $-1.25 \pm 0.02$ & $-2.10 \pm 0.02$ & $-1.18 \pm 0.01$ & $-2.05 \pm 0.02$ \\
\hline
\end{tabular}

as worse agreement between experimental measurements and simulation results than in the Shaping model case. This indicates that the shaping model implemented in GBS improves the description of SOL plasma turbulence by taking into account the impact of elongation and triangularity. At the same time, for the circular discharge \#55391, the Circular model is in slightly better agreement with the experiment than the Shaping model. We also observe that $\chi$ is larger for the two-dimensional model than for the Shaping model, indicating that the latter is in better agreement with the

TABLE XIV. Level of agreement between simulations and experiments and accuracy parameter with respect to the $j_{\text {sat }}$ spectral-slopes.

\begin{tabular}{|c|c|c|c|c|c|c|}
\hline & \multicolumn{2}{|c|}{ \#54147 } & \multicolumn{2}{|c|}{ \#55391 } & \multicolumn{2}{|c|}{ \#55394 } \\
\hline & $R_{\alpha_{j s a t}}$ & $S_{\alpha_{j s a t}}$ & $R_{\alpha_{j s a t}}$ & $S_{\alpha_{j s a t}}$ & $R_{\alpha_{j s a t}}$ & $S_{\alpha_{j_{s a t}}}$ \\
\hline Shaping model & 0.89 & 0.89 & 0.23 & 0.86 & 0.98 & 0.86 \\
\hline Circular model & 1.00 & 0.89 & 0.03 & 0.86 & 0.99 & 0.86 \\
\hline $2 \mathrm{D}$ model & 1.00 & 0.90 & 0.40 & 0.87 & 1.00 & 0.87 \\
\hline
\end{tabular}

experimental measurements. On the other hand, by comparing the results of the $2 \mathrm{D}$ and Circular models, it is not possible to identify a clear trend, as the $2 \mathrm{D}$ model seems to perform better for the \#54147 plasma discharge and worse for the \#55391 and \#55394 discharges. Nevertheless, we speculate that better agreement between two-dimensional simulations and experimental measurements could be obtained by considering different closures for the parallel terms (as shown, for example, in Refs. 30 and 31) or by including the hot ion dynamics.

TABLE XV. Level of agreement between simulations and experiments and accuracy parameter with respect to the $V_{f l}$ spectral-slopes.

\begin{tabular}{|c|c|c|c|c|c|c|}
\hline & \multicolumn{2}{|c|}{ \#54147 } & \multicolumn{2}{|c|}{ \#55391 } & \multicolumn{2}{|c|}{ \#55394 } \\
\hline & $R_{\alpha_{v_{f l}}}$ & $S_{\alpha_{V_{f f}}}$ & $R_{\alpha_{v_{f l}}}$ & $S_{\alpha_{V_{f l}}}$ & $R_{\alpha_{V_{f l}}}$ & $S_{\alpha_{V_{f l}}}$ \\
\hline Shaping model & 0.53 & 0.81 & 1.00 & 0.87 & 1.00 & 0.88 \\
\hline Circular model & 0.74 & 0.80 & 0.98 & 0.86 & 1.00 & 0.88 \\
\hline 2D model & 0.03 & 0.84 & 0.03 & 0.90 & 0.46 & 0.91 \\
\hline
\end{tabular}


TABLE XVI. Combined metric $\chi$ and quality factor $Q$, considering the ten observables discussed in Sec. V.

\begin{tabular}{|c|c|c|c|c|c|c|}
\hline & \multicolumn{2}{|c|}{ \#54147 } & \multicolumn{2}{|c|}{ \#55391 } & \multicolumn{2}{|c|}{ \#55394 } \\
\hline & $\chi$ & $Q$ & $X$ & $Q$ & $\chi$ & $Q$ \\
\hline Shaping model & 0.45 & 3.72 & 0.48 & 4.02 & 0.45 & 4.16 \\
\hline Circular model & 0.64 & 3.83 & 0.42 & 4.06 & 0.63 & 4.21 \\
\hline 2D model & 0.59 & 4.11 & 0.56 & 4.33 & 0.75 & 4.43 \\
\hline
\end{tabular}

In summary, the study of the validation table shows that, while equilibrium profiles and the nature of the main instabilities are reasonably well described by the Shaping model, some turbulence details are not well modeled. This is particularly true for $j_{\text {sat }}$ fluctuations, which are underestimated by the simulations by approximately a factor of 2 .

Since the uncertainties for all the observables are rather small (with some specific exceptions) and all the observables are at the second level of the validation hierarchy, $\mathrm{Q}$ is about constant in our comparison. In particular, $Q \approx 4$, which is close to the maximum value $Q=5$ that can be obtained by considering ten observables with $H=0.5$. The $Q$ values reported in Table XVI can be compared with the $Q$ that would be obtained by comparing exclusively the agreement of the experimental and simulation SOL gradient scale lengths, that is, $Q \leq 1.5$. Finally, we note that, to investigate the sensitivity of $\chi$ on $d_{0}$ and $\lambda$, we also computed $\chi$ for $d_{0}=0.5,1.0,1.5$ and $\lambda=0.5$ and for $d_{0}=1.0$ and $\lambda=0.1,0.5,1.0$. We find that, while the exact value of $\chi$ depends on $d_{0}$ and $\lambda$, the conclusions obtained in this work are not affected by varying these parameters.

The rigorous validation exercise presented herein shows that the model introduced in Ref. 26 constitutes an effective improvement of our capabilities to describe SOL turbulence in elongated magnetic equilibria with finite triangularity. This increases the reliability of our numerical results, showing that GBS simulations are a suitable tool for investigating SOL plasma turbulence in limited noncircular magnetic geometries. However, while quantities of primary importance, such as the density and temperature scale lengths, are well represented by our model, significant discrepancies persist in other observables, in particular for what concerns the level of fluctuations and the PSDs. These discrepancies can be related to, e.g., the use of a fluid model or the coupling of the SOL dynamics with the core plasma. We expect that the improvement of our model and a series of rigorous validation exercises will allow us to identify the reasons behind these discrepancies.

The present work provides a concrete example of application of the validation methodology introduced by Ricci et al. in Refs. 24 and 45 to SOL plasma turbulence in tokamaks, showing that this methodology is able to discriminate among different models and assess the most suited to describing the experimental measurements. On the other hand, it is much more difficult to judge a single model in absolute terms, since establishing if the observed level of agreement is acceptable or not depends on the specific purposes of the simulations. It should be noted that, while sophisticated methodologies for estimating $\Delta e_{i, j}$ and $\Delta s_{i, j}$ have been developed in the past few years, such as Bayesian analysis, ${ }^{67}$ multifidelity Monte Carlo estimations, ${ }^{68}$ or the stochastic response surface methodology, ${ }^{69,70}$ these methodologies are extremely challenging to apply in the context of SOL plasma turbulence simulations. The methodology used in this publication can be considered a compromise between an easy-to-use validation technique and more rigorous, but also more cumbersome, approaches.

\section{ACKNOWLEDGMENTS}

The simulations presented herein were carried out in part at the Swiss National Supercomputing Center (CSCS) under Project IDs s655 and s718 and in part on the CINECA Marconi supercomputer within the framework of the GBSSOL and GBSedge projects. This work was carried out within the framework of the EUROfusion Consortium and received funding from the Euratom research and training programme 2014-2018 and 2019-2020 under Grant Agreement No. 633053, from the RCUK Energy Programme under Grant No. EP/P012450/1, and from the U.S. Department of Energy under Grant No. DE-SC0010529. The views and opinions expressed herein do not necessarily reflect those of the European Commission.

\section{REFERENCES}

${ }^{1}$ W. L. Oberkampf and T. G. Trucano, "Verification and validation in computational fluid dynamics,” Prog. Aerosp. Sci. 38, 209-272 (2002).

${ }^{2}$ W. L. Oberkampf and C. J. Roy, Verification and Validation in Scientific Computing (Cambridge University Press, New York, NY, USA, 2010).

${ }^{3}$ P. J. Roache, Verification and Validation in Computational Science and Engineering (Hermosa Publishers, Albuquerque, NM, USA, 1998).

${ }^{4}$ American Institute of Aeronautics \& Astronautics, Guide for the Verification and Validation of Computational Fluid Dynamics Simulations, G-077-1998 (American Institute of Aeronautics \& Astronautics, 1999).

${ }^{5}$ A. M. Dimits, G. Bateman, M. A. Beer, B. I. Cohen, W. Dorland, G. W. Hammett, C. Kim, J. E. Kinsey, M. Kotschenreuther, A. H. Kritz, L. L. Lao, J. Mandrekas, W. M. Nevins, S. E. Parker, A. J. Redd, D. E. Shumaker, R. Sydora, and J. Weiland, "Comparisons and physics basis of tokamak transport models and turbulence simulations," Phys. Plasmas 7, 969 (2000).

${ }^{6}$ P. Ricci, J. U. Brackbill, W. Daughton, and G. Lapenta, "Collisionless magnetic reconnection in the presence of a guide field," Phys. Plasmas 11, 4102 (2004).

${ }^{7}$ G. L. Falchetto, B. D. Scott, P. Angelino, A. Bottino, T. Dannert, V. Grandgirard, S. Janhunen, F. Jenko, S. Jolliet, A. Kendl, B. F. McMillan, V. Naulin, A. H. Nielsen, M. Ottaviani, A. G. Peeters, M. J. Pueschel, D. Reiser, T. T. Ribeiro, and M. Romanelli, “The European turbulence code benchmarking effort: Turbulence driven by thermal gradients in magnetically confined plasmas," Plasma Phys. Controlled Fusion 50, 124015 (2008).

${ }^{8}$ M. M. Turner, A. Derzsi, Z. Donko, D. Eremin, S. J. Kelly, T. Lafleur, and T. Mussenbrock, "Simulation benchmarks for low-pressure plasmas: Capacitive discharges," Phys. Plasmas 20, 013507 (2013).

${ }^{9}$ R. V. Bravenec, Y. Chen, J. Candy, W. Wan, and S. Parker, "A verification of the gyrokinetic microstability codes GEM, GYRO, and GS2," Phys. Plasmas 20, 104506 (2013).

${ }^{10} \mathrm{~J}$. Brackbill, "On energy and momentum conservation in particle-in-cell plasma simulation," J. Comput. Phys. 317, 405-427 (2016).

${ }^{11} \mathrm{P}$. Roache, Fundamentals of Verification and Validation (Hermosa Publication, 2009).

${ }^{12}$ F. Riva, P. Ricci, F. D. Halpern, S. Jolliet, J. Loizu, and A. Mosetto, "Verification methodology for plasma simulations and application to a scrape-off layer turbulence code," Phys. Plasmas 21, 062301 (2014).

${ }^{13}$ B. D. Dudson, J. Madsen, J. Omotani, P. Hill, L. Easy, and M. Løiten, "Verification of BOUT ++ by the method of manufactured solutions," Phys. Plasmas 23, 062303 (2016).

${ }^{14}$ P. Tamain, H. Bufferand, G. Ciraolo, C. Colin, D. Galassi, P. Ghendrih, F. Schwander, and E. Serre, "The TOKAM3X code for edge turbulence fluid simulations of tokamak plasmas in versatile magnetic geometries," J. Comput. Phys. 321, 606-623 (2016).

${ }^{15}$ F. Riva, C. F. Beadle, and P. Ricci, "A methodology for the rigorous verification of particle-in-cell simulations,” Phys. Plasmas 24, 055703 (2017). 
${ }^{16}$ A. Stegmeir, D. Coster, A. Ross, O. Maj, K. Lackner, and E. Poli, "GRILLIX: A 3D turbulence code based on the flux-coordinate independent approach," Plasma Phys. Controlled Fusion 60, 035005 (2018).

${ }^{17}$ P. W. Terry, M. Greenwald, J.-N. Leboeuf, G. R. McKee, D. R. Mikkelsen, W. M. Nevins, D. E. Newman, and D. P. Stotler, "Validation in fusion research: Towards guidelines and best practices," Phys. Plasmas 15, 062503 (2008).

${ }^{18} \mathrm{M}$. Greenwald, "Verification and validation for magnetic fusion," Phys. Plasmas 17, 058101 (2010).

${ }^{19} \mathrm{C}$. Holland, "Validation metrics for turbulent plasma transport," Phys. Plasmas 23, 060901 (2016).

${ }^{20}$ S. J. Freethy, T. Görler, A. J. Creely, G. D. Conway, S. S. Denk, T. Happel, C. Koenen, P. Hennequin, A. E. White, and ASDEX Upgrade Team, "Validation of gyrokinetic simulations with measurements of electron temperature fluctuations and density-temperature phase angles on ASDEX Upgrade," Phys. Plasmas 25, 055903 (2018).

${ }^{21} \mathrm{~A}$. E. White, "Validation of nonlinear gyrokinetic transport models using turbulence measurements," J. Plasma Phys. 85, 925850102 (2019).

${ }^{22}$ A. Ho, J. Citrin, F. Auriemma, C. Bourdelle, F. J. Casson, H.-T. Kim, P. Manas, G. Szepesi, H. Weisen, and JET Contributors. "Application of Gaussian process regression to plasma turbulent transport model validation via integrated modelling," Nucl. Fusion 59, 056007 (2019).

${ }^{23} \mathrm{~F}$. Riva, "Verification and validation procedures with applications to plasmaedge turbulence simulations," Ph.D. thesis (SB, Lausanne, 2017).

${ }^{24}$ P. Ricci, C. Theiler, a Fasoli, I. Furno, K. Gustafson, D. Iraji, and J. Loizu, "Methodology for turbulence code validation: Quantification of simulationexperiment agreement and application to the TORPEX experiment," Phys. Plasmas 18, 032109 (2011).

${ }^{25}$ F. Hofmann, J. B. Lister, W. Anton, S. Barry, R. Behn, S. Bernel, G. Besson, F. Buhlmann, R. Chavan, M. Corboz, M. J. Dutch, B. P. Duval, D. Fasel, A. Favre, S. Franke, A. Heym, A. Hirt, C. Hollenstein, P. Isoz, B. Joye, X. Llobet, J. C. Magnin, B. Marletaz, P. Marmillod, Y. Martin, J. M. Mayor, J. M. Moret, C. Nieswand, P. J. Paris, A. Perez, Z. A. Pietrzyk, R. A. Pitts, A. Pochelon, R. Rage, O. Sauter, G. Tonetti, M. Q. Tran, F. Troyon, D. J. Ward, and H. Weisen, "Creation and control of variably shaped plasmas in TCV," Plasma Phys. Controlled Fusion 36, B277-B287 (1994).

${ }^{26}$ F. Riva, E. Lanti, S. Jolliet, and P. Ricci, "Plasma shaping effects on tokamak scrape-off layer turbulence," Plasma Phys. Controlled Fusion 59, 035001 (2017).

${ }^{27}$ Y. Camenen, A. Pochelon, R. Behn, A. Bottino, A. Bortolon, S. Coda, A. Karpushov, O. Sauter, G. Zhuang, and TCV Team, "Impact of plasma triangularity and collisionality on electron heat transport in TCV L-mode plasmas," Nucl. Fusion 47, 510-516 (2007).

${ }^{28}$ J. Horacek, R. A. Pitts, J. Adamek, G. Arnoux, J.-G. Bak, S. Brezinsek, M. Dimitrova, R. J. Goldston, J. P. Gunn, J. Havlicek, S.-H. Hong, F. Janky, B. LaBombard, S. Marsen, G. Maddaluno, L. Nie, V. Pericoli, T. Popov, R. Panek, D. Rudakov, J. Seidl, D. S. Seo, M. Shimada, C. Silva, P. C. Stangeby, B. Viola, P. Vondracek, H. Wang, G. S. Xu, and Y. Xu, "Multi-machine scaling of the main SOL parallel heat flux width in tokamak limiter plasmas," Plasma Phys. Controlled Fusion 58, 074005 (2016).

${ }^{29}$ J. P. Graves, J. Horacek, R. A. Pitts, and K. I. Hopcraft, "Self-similar density turbulence in the TCV tokamak scrape-off layer," Plasma Phys. Controlled Fusion 47, L1-L9 (2005).

${ }^{30}$ O. E. Garcia, J. Horacek, R. A. Pitts, A. H. Nielsen, W. Fundamenski, J. P. Graves, V. Naulin, and J. J. Rasmussen, "Interchange turbulence in the TCV scrape-off layer," Plasma Phys. Controlled Fusion 48, L1-L10 (2006).

${ }^{31}$ O. Garcia, J. Horacek, R. Pitts, A. Nielsen, W. Fundamenski, V. Naulin, and J. J. Rasmussen, "Fluctuations and transport in the TCV scrape-off layer," Nucl. Fusion 47, 667-676 (2007).

${ }^{32}$ F. Nespoli, I. Furno, F. Halpern, B. Labit, J. Loizu, P. Ricci, and F. Riva, "Nonlinear simulations of the TCV scrape-off layer," Nucl. Mater. Energy 12, 1205-1208 (2017)

${ }^{33}$ C. K. Tsui, J. A. Boedo, J. R. Myra, B. Duval, B. Labit, C. Theiler, N. Vianello, W. A. J. Vijvers, H. Reimerdes, S. Coda, O. Février, J. R. Harrison, J. Horacek, B. Lipschultz, R. Maurizio, F. Nespoli, U. Sheikh, K. Verhaegh, and N. Walkden, "Filamentary velocity scaling validation in the TCV tokamak," Phys. Plasmas 25, 072506 (2018).
${ }^{34}$ S. I. Braginskii, "Transport processes in a plasma," Rev. Plasma Phys. 1, 205 (1965).

${ }^{35}$ A. Zeiler, J. F. Drake, and B. Rogers, "Nonlinear reduced Braginskii equations with ion thermal dynamics in toroidal plasma," Phys. Plasmas 4, 2134 (1997).

${ }^{36}$ L. Easy, F. Militello, J. Omotani, B. Dudson, E. Havlíčková, P. Tamain, V. Naulin, and A. H. Nielsen, "Three dimensional simulations of plasma filaments in the scrape off layer: A comparison with models of reduced dimensionality," Phys. Plasmas 21, 122515 (2014).

${ }^{37}$ O. E. Garcia, V. Naulin, A. H. Nielsen, and J. J. Rasmussen, "Computations of intermittent transport in scrape-off layer plasmas," Phys. Rev. Lett. 92, 165003 (2004).

${ }^{38}$ P. Ricci, F. D. Halpern, S. Jolliet, J. Loizu, A. Mosetto, A. Fasoli, I. Furno, and C. Theiler, "Simulation of plasma turbulence in scrape-off layer conditions: The GBS code, simulation results and code validation," Plasma Phys. Controlled Fusion 54, 124047 (2012).

${ }^{39}$ A. Nielsen, G. Xu, J. Madsen, V. Naulin, J. Juul Rasmussen, and B. Wan, "Simulation of transition dynamics to high confinement in fusion plasmas," Phys. Lett. A 379, 3097-3101 (2015).

${ }^{40}$ F. D. Halpern, P. Ricci, S. Jolliet, J. Loizu, J. Morales, A. Mosetto, F. Musil, F. Riva, T. M. Tran, and C. Wersal, "The GBS code for tokamak scrape-off layer simulations," J. Comput. Phys. 315, 388-408 (2016).

${ }^{41}$ B. D. Dudson and J. Leddy, "Hermes: Global plasma edge fluid turbulence simulations," Plasma Phys. Controlled Fusion 59, 054010 (2017).

${ }^{42}$ B. Zhu, M. Francisquez, and B. N. Rogers, "GDB: A global 3D two-fluid model of plasma turbulence and transport in the tokamak edge," Comput. Phys. Commun. 232, 46-58 (2018)

${ }^{43}$ F. D. Halpern, J. Horacek, R. A. Pitts, and P. Ricci, "A theoretical interpretation of the main scrape-off layer heat-flux width scaling for tokamak inner-wall limited plasmas," Plasma Phys. Controlled Fusion 58, 084003 (2016).

${ }^{44}$ J. A. Boedo, N. Crocker, L. Chousal, R. Hernandez, J. Chalfant, H. Kugel, P. Roney, and J. Wertenbaker, "Fast scanning probe for the NSTX spherical tokamak," Rev. Sci. Instrum. 80, 123506 (2009).

${ }^{45}$ P. Ricci, F. Riva, C. Theiler, A. Fasoli, I. Furno, F. D. Halpern, and J. Loizu, "Approaching the investigation of plasma turbulence through a rigorous verification and validation procedure: A practical example," Phys. Plasmas 22, 055704 (2015).

${ }^{46}$ I. Furno, F. Avino, A. Bovet, A. Diallo, A. Fasoli, K. Gustafson, D. Iraji, B. Labit, J. Loizu, S. H. Müller, G. Plyushchev, M. Podestà, F. M. Poli, P. Ricci, and C. Theiler, "Plasma turbulence, suprathermal ion dynamics and code validation on the basic plasma physics device TORPEX," J. Plasma Phys. 81, 345810301 (2015).

${ }^{47}$ P. Ricci, C. Theiler, A. Fasoli, I. Furno, B. Labit, S. H. Muller, M. Podesta, and F. M. Poli, "Langmuir probe-based observables for plasma-turbulence code validation and application to the TORPEX basic plasma physics experiment," Phys. Plasmas 16, 055703 (2009).

${ }^{48} \mathrm{~B}$. N. Rogers and P. Ricci, "Low-frequency turbulence in a linear magnetized plasma," Phys. Rev. Lett. 104, 225002 (2010).

${ }^{49} \mathrm{P}$. Ricci and B. N. Rogers, "Turbulence phase space in simple magnetized toroidal plasmas," Phys. Rev. Lett. 104, 145001 (2010).

${ }^{50}$ P. Paruta, P. Ricci, F. Riva, C. Wersal, C. Beadle, and B. Frei, "Simulation of plasma turbulence in the periphery of diverted tokamak by using the GBS code," Phys. Plasmas 25, 112301 (2018).

${ }^{51}$ D. A. Russell, D. A. D’Ippolito, and J. R. Myra, "On relaxing the Boussinesq approximation in scrape-off layer turbulence (SOLT) model simulations," in 54th Annual Meeting of the APS Division of Plasma Physics, Bulletin of the American Physical Society, Providence, RI (2012), Vol. 57, Paper No. BP8.159.

${ }^{52} \mathrm{G}$. Q. Yu, S. I. Krasheninnikov, and P. N. Guzdar, "Two-dimensional modelling of blob dynamics in tokamak edge plasmas," Phys. Plasmas 13, 042508 (2006).

${ }^{53}$ K. Bodi, G. Ciraolo, P. Ghendrih, F. Schwander, E. Serre, and P. Tamain, "Impact of the Boussinesq approximation in tokamak scrape-off layer turbulence," in 38th EPS Conference on Plasma Physics, Strasbourg, France (2011), Paper No. P1.121.

${ }^{54} \mathrm{~A}$. Ross, A. Stegmeir, and D. Coster, "Effect of the Boussinesq approximation: Turbulence studies with GRILLIX in slab geometry," Contrib. Plasma Phys. 58, 478-483 (2018).

${ }^{55}$ J. Loizu, P. Ricci, F. D. Halpern, and S. Jolliet, "Boundary conditions for plasma fluid models at the magnetic presheath entrance," Phys. Plasmas 19, 122307 (2012). 
${ }^{56}$ A. Mosetto, F. D. Halpern, S. Jolliet, J. Loizu, and P. Ricci, "Finite ion temperature effects on scrape-off layer turbulence," Phys. Plasmas 22, 012308 (2015).

${ }^{57}$ S. Jolliet, F. D. Halpern, J. Loizu, A. Mosetto, and P. Ricci, "Aspect ratio effects on limited scrape-off layer plasma turbulence," Phys. Plasmas 21, 022303 (2014).

${ }^{58}$ S. Coda, J. Ahn, R. Albanese, S. Alberti, E. Alessi, S. Allan, H. Anand, G. Anastassiou, Y. Andrèbe, C. Angioni, M. Ariola, M. Bernert, M. Beurskens, W. Bin, P. Blanchard, T. Blanken, J. Boedo, T. Bolzonella, F. Bouquey, F. Braunmüller, H. Bufferand, P. Buratti, G. Calabró, Y. Camenen, D. Carnevale, F. Carpanese, F. Causa, R. Cesario, I. Chapman, O. Chellai, D. Choi, C. Cianfarani, G. Ciraolo, J. Citrin, S. Costea, F. Crisanti, N. Cruz, A. Czarnecka, J. Decker, G. De Masi, G. De Tommasi, D. Douai, M. Dunne, B. Duval, T. Eich, S. Elmore, B. Esposito, M. Faitsch, A. Fasoli, N. Fedorczak, F. Felici, O. Février, O. Ficker, S. Fietz, M. Fontana, L. Frassinetti, I. Furno, S. Galeani, A. Gallo, C. Galperti, S. Garavaglia, I. Garrido, B. Geiger, E. Giovannozzi, M. Gobbin, T. Goodman, G. Gorini, M. Gospodarczyk, G. Granucci, J. Graves, R. Guirlet, A. Hakola, C. Ham, J. Harrison, J. Hawke, P. Hennequin, B. Hnat, D. Hogeweij, J.-P. Hogge, C. Honoré, C. Hopf, J. Horáček, Z. Huang, V. Igochine, P. Innocente, C. Ionita Schrittwieser, H. Isliker, R. Jacquier, A. Jardin, J. Kamleitner, A. Karpushov, D. Keeling, N. Kirneva, M. Kong, M. Koubiti, J. Kovacic, A. Krämer-Flecken, N. Krawczyk, O. Kudlacek, B. Labit, E. Lazzaro, H. Le, B. Lipschultz, X. Llobet, B. Lomanowski, V. Loschiavo, T. Lunt, P. Maget, E. Maljaars, A. Malygin, M. Maraschek, C. Marini, P. Martin, Y. Martin, S. Mastrostefano, R. Maurizio, M. Mavridis, D. Mazon, R. McAdams, R. McDermott, A. Merle, H. Meyer, F. Militello, I. Miron, P. Molina Cabrera, J.-M. Moret, A. Moro, D. Moulton, V. Naulin, F. Nespoli, A. Nielsen, M. Nocente, R. Nouailletas, S. Nowak, T. Odstrčil, G. Papp, R. Paprok, A. Pau, G. Pautasso, V. Pericoli Ridolfini, P. Piovesan, C. Piron, T. Pisokas, L. Porte, M. Preynas, G. Ramogida, C. Rapson, J. Juul Rasmussen, M. Reich, H. Reimerdes, C. Reux, P. Ricci, D. Rittich, F. Riva, T. Robinson, S. Saarelma, F. SaintLaurent, O. Sauter, R. Scannell, C. Schlatter, B. Schneider, P. Schneider, R. Schrittwieser, F. Sciortino, M. Sertoli, U. Sheikh, B. Sieglin, M. Silva, J. Sinha, C. Sozzi, M. Spolaore, T. Stange, T. Stoltzfus-Dueck, P. Tamain, A. Teplukhina, D. Testa, C. Theiler, A. Thornton, L. Tophøj, M. Tran, C. Tsironis, C. Tsui, A. Uccello, S. Vartanian, G. Verdoolaege, K. Verhaegh, L. Vermare, N. Vianello, W. Vijvers, L. Vlahos, N. Vu, N. Walkden, T. Wauters, H. Weisen, M. Wischmeier, P. Zestanakis, and M. Zuin, "Overview of the TCV tokamak program: Scientific progress and facility upgrades," Nucl. Fusion 57, 102011 (2017).
${ }^{59}$ J.-M. Moret, B. Duval, H. Le, S. Coda, F. Felici, and H. Reimerdes, "Tokamak equilibrium reconstruction code LIUQE and its real time implementation," Fusion Eng. Des. 91, 1-15 (2015).

${ }^{60}$ C. K. Tsui, J. A. Boedo, F. D. Halpern, J. Loizu, F. Nespoli, J. Horacek, B. Labit, J. Morales, H. Reimerdes, P. Ricci, C. Theiler, S. Coda, B. P. Duval, and I. Furno, "Poloidal asymmetry in the narrow heat flux feature in the TCV scrape-off layer," Phys. Plasmas 24, 062508 (2017).

${ }^{61}$ A. Mosetto, F. D. Halpern, S. Jolliet, J. Loizu, and P. Ricci, "Turbulent regimes in the tokamak scrape-off layer," Phys. Plasmas 20, 092308 (2013).

${ }^{62}$ C. K. Tsui, J. A. Boedo, and P. C. Stangeby, "Accounting for Debye sheath expansion for proud Langmuir probes in magnetic confinement fusion plasmas,” Rev. Sci. Instrum. 89, 013505 (2018).

${ }^{63}$ F. Halpern and P. Ricci, "Velocity shear, turbulent saturation, and steep plasma gradients in the scrape-off layer of inner-wall limited tokamaks," Nucl. Fusion 57, 034001 (2017).

${ }^{64}$ F. Nespoli, B. Labit, I. Furno, J. Horacek, C. Tsui, J. Boedo, R. Maurizio, H. Reimerdes, C. Theiler, P. Ricci, F. Halpern, U. Sheikh, K. Verhaegh, R. Pitts, and F. Militello, "Understanding and suppressing the near scrape-off layer heat flux feature in inboard-limited plasmas in TCV," Nucl. Fusion 57, 126029 (2017).

${ }^{65}$ F. Riva, L. Milanese, and P. Ricci, "Uncertainty propagation by using spectral methods: A practical application to a two-dimensional turbulence fluid model," Phys. Plasmas 24, 102302 (2017).

${ }^{66}$ F. Riva, N. Vianello, M. Spolaore, P. Ricci, R. Cavazzana, L. Marrelli, and S. Spagnolo, "Three-dimensional simulations of plasma turbulence in the RFXmod scrape-off layer and comparison with experimental measurements," Phys. Plasmas 25, 022305 (2018)

${ }^{67}$ W. L. Oberkampf, T. G. Trucano, and C. Hirsch, "Verification, validation, and predictive capability in computational engineering and physics," Appl. Mech. Rev, 57, 345 (2004)

${ }^{68}$ B. Peherstorfer, K. Willcox, and M. Gunzburger, "Optimal model management for multifidelity Monte Carlo estimation," SIAM J. Sci. Comput. 38, A3163-A3194 (2016)

${ }^{69}$ S. S. Isukapalli, A. Roy, and P. G. Georgopoulos, "Stochastic Response Surface Methods (SRSMs) for uncertainty propagation: application to environmental and biological systems," Risk Anal. 18, 351-363 (1998).

${ }^{70} \mathrm{R}$. Myers, D. Montgomery, and C. Anderson-Cook, Response Surface Methodology: Process and Product Optimization Using Designed Experiments, Wiley Series in Probability and Statistics (Wiley, 2016). 